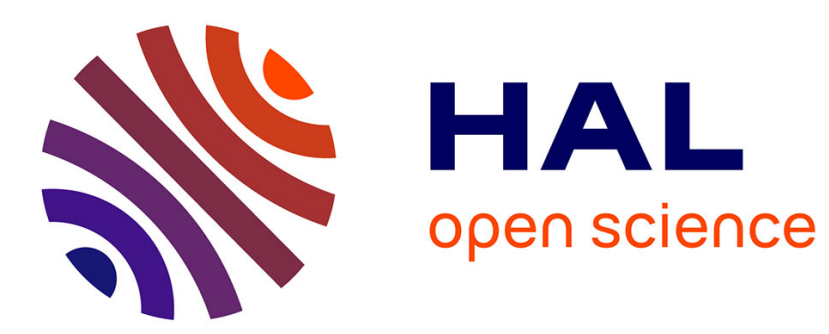

\title{
Equilibria with increasing returns: sufficient conditions on bounded production allocations
}

\author{
Jean-Marc Bonnisseau, Alexandrine Jamin
}

\section{To cite this version:}

Jean-Marc Bonnisseau, Alexandrine Jamin. Equilibria with increasing returns: sufficient conditions on bounded production allocations. 2005. halshs-00194881

\section{HAL Id: halshs-00194881 \\ https://shs.hal.science/halshs-00194881}

Submitted on 7 Dec 2007

HAL is a multi-disciplinary open access archive for the deposit and dissemination of scientific research documents, whether they are published or not. The documents may come from teaching and research institutions in France or abroad, or from public or private research centers.
L'archive ouverte pluridisciplinaire HAL, est destinée au dépôt et à la diffusion de documents scientifiques de niveau recherche, publiés ou non, émanant des établissements d'enseignement et de recherche français ou étrangers, des laboratoires publics ou privés. 

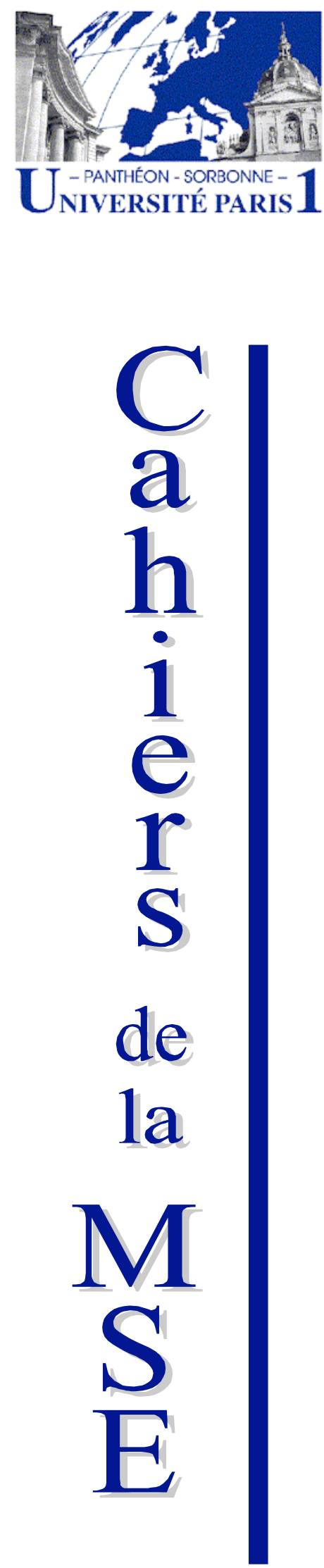

Equilibria with increasing returns : sufficient conditions on bounded production allocations

Jean-Marc BonNisseau, CERMSEM

Alexandrine JAMIN, CERMSEM

2005.45 


\title{
Equilibria with Increasing Returns: Sufficient Conditions on Bounded Production Allocations
}

\author{
J.-M. Bonnisseau, A. Jamin \\ CERMSEM CNRS-UMR 8095, \\ Maison des Sciences Économiques \\ 106-112 Boulevard de l'Hôpital \\ 75647 Paris cedex 13, France
}

\begin{abstract}
This paper deals with the existence of marginal pricing equilibria or equilibria with general pricing rules in an economy with increasing returns to scale or more general types of non convexities in production. Its main contribution is to posit the bounded loss and survival assumptions on a bounded subset of production allocations. Furthermore, the free-disposal assumption is weaken, which allows to consider non positive prices. Finally, we also provide an existence result for a quasi-equilibria, when the survival assumption is weaken on the attainable allocations.
\end{abstract}

Keywords General economic equilibrium , increasing returns, general pricing rules, bounded losses, marginal pricing rule, free disposal.

\section{Introduction}

Since Guesnerie (1975), many research works have addressed the existence problem of equilibria in economies with increasing returns or exhibiting more general types of non-convexities in the production sector. The failure of the competitive mechanism in this situation has led to reconsider the firms behaviour. Various alternatives were proposed in former economic literature, that have been formalized in the general equilibrium theory framework.

Email addresses: Jean-Marc.Bonnisseau@univ-paris1.fr (J.-M. Bonnisseau), Alexandrine. Jamin@univ-paris1.fr (A. Jamin). 
One of the most important is the marginal cost theory. Following Hotelling (1938), economic efficiency could be achieved only if every commodity is sold at marginal cost. Formally, firms are supposed to minimize their costs and set their selling prices equal to marginal cost. This implicitly supposes that there is a cost function associated with every available technology, and that it is differentiable. To allow for non smooth production sets, this rule was generalized and called the marginal pricing rule: firms follow the marginal pricing rule if they fulfill the first order necessary condition for their profit maximization, in the precise mathematical sense formalized by Clarke's normal cone [see Cornet (1989)].

Another theory then followed from the will to unify the existing results and to allow for a larger diversity for firms behaviours in an economy. In this theory, firms are supposed to follow a general pricing rule, which can be the average pricing rule, the voluntary pricing rule, a Ramsey-Boiteux like pricing rule or many others [see Dierker et al. (1985), Dehez and Drèze (1988a, 1988b), Bonnisseau and Cornet (1988)].

There is an important gap between these two theories. Indeed, while more assumptions are required in existence results for general pricing rules (the bounded loss assumption, for instance), the existence proofs for marginal pricing equilibria involve more sophisticated mathematical techniques from differential topology. However, this gap is bridged by Bonnisseau (1992) who proves that, under several standard assumptions, a general pricing rule with bounded losses can be found, that coincides with the marginal pricing rule on the attainable set. This allows us to derive the existence of a marginal pricing equilibrium from the existence of an equilibrium for general pricing rules with bounded losses.

The purpose of this paper is to weaken three crucial assumptions on production allocations, namely the boundedness, the survival and the bounded losses assumptions. To present these assumptions, we first define the t-attainable production allocations for a real number $t \geq 0$, which are the production allocations that become attainable when $t$ units of a reference commodity bundle (which can be seen as a specie) are injected in the economy.

The boundedness assumption ensures that, for every $t \geq 0$, the set of the $t$ attainable production allocations is bounded. The bounded losses assumption states that the losses of the firms are bounded below when they follow their pricing rules. Finally, the survival assumption states that, for every $t \geq 0$, and every $t$-attainable production allocation, if all the firms set the same price according to the pricing rule, then the global profit (or loss) plus the value of the $t$ units of the reference commodity bundle is strictly greater than the consumer's subsistence level. 
A major weakness of these assumptions leads in the fact that they involve very large production whereas the equilibrium allocation always lies in the set of attainable allocations. So, we need asymptotic properties of the production sets although the interesting part is bounded. Note that the usual convexity assumption shares the same weakness.

In order to remain as close as possible to the attainable allocations, which are the relevant ones from an economic point of view, we propose here to limit the boundedness, the bounded losses and the survival assumptions to a bounded subset of production allocations. More precisely, we assume that the set of $t_{0}$-attainable allocations is bounded for some nonnegative real number $t_{0}$. Then, we assume that the survival assumption holds true on the set of strictly $t_{0}$-attainable allocations, that is the set of allocations $t$-attainable for some $t<t_{0}$. Finally, we state a bounded losses-like assumption on the set of production allocations, which are $t_{1}$-attainable but not strictly $t_{0}$-attainable. The difference between $t_{1}$ and $t_{0}$ is a measure of the nonconvexity of the production sets. If the set of $t_{0}$-attainable allocation is convex, then $t_{1}=t_{0}$.

In the particular case of the loss-free pricing rule, we only need to assume that the set of attainable production allocations is bounded. So, we recover a result of Bonnisseau and Cornet (1988) within a general framework whereas a particular argument was needed before.

The main interest of this result comes from the fact that it can be used to study perturbed economy, when this perturbation affects the pricing rules. Thus, if an economy satisfies the necessary conditions for the existence of an equilibrium, every close enough economy satisfies them also. Indeed, since we only need to checks some inequalities on compact sets, we can expect that the inequalities remain true even for sufficiently small perturbations.

Another interest comes from the applications to the economies with infinitely many commodities. Indeed, since we cannot expect to get a Pareto optimal equilibrium allocation, the only way to prove the existence of an equilibrium is to consider a finite dimensional approximation of the commodity space, and, then to do a limit argument. But, it remains to prove that an equilibrium exists in the finite dimensional auxiliary economies. For this, starting from assumptions on the infinite dimensional original economy, we can again expect that the approximate economy satisfy the necessary conditions for the existence of an equilibrium if the commodity space is large enough.

Another contribution of this paper is a weakening of the free-disposal assumption. Instead of considering the standard positive orthantt, we suppose the existence of a free-disposal cone, which is a pointed closed cone with a nonempty interior. An interesting consequence is that we allow the firms to set negative prices for some commodities at some production. This feature may 
be useful in approximating infinite dimensional economies or when we want to incorporate some strategic aspect in the behavior of the firms by considering imperfect competition à la Negishi [see Dehez et al. (2003)].

This paper is organized as follows. In the next section, we present the model and posit the standard assumptions that describe the general framework. In section 3 we state our main existence result (Theorem 1), which proof rests on a generalization of the fundamental result of Debreu (1956), Gale (1955) and Nikaidô (1956) (Theorem 2). In section 4, thanks to the unifying result in Bonnisseau (1992), we deduce from Theorem 1 the existence of marginal pricing equilibria (Theorem 3). Finally, we give in section 5 an existence result for quasi-equilibria (Theorem 4) when the survival assumption is no longer supposed satisfied on the set of the attainable production allocations. The Appendix gathers the proofs of all the lemmata stated along the paper.

\section{The model and the assumptions}

The commodity space is a $\ell$-dimensional Euclidean space $L$. We denote by $\omega \in L$ the vector of total initial endowments of the economy.

We consider a finite number $n$ of firms (indexed by $j=1, \ldots, n$ ). The technological possibilities of firms $j$ are represented by its production set $Y_{j} \subset L$.

Assumption (P)

For every $j, Y_{j}$ is a nonempty, closed subset of $L$, different from $L$. There exists a closed, convex, pointed cone $D$ of vertex 0 in $L$ with a nonempty interior such that, for every $j, Y_{j}$ satisfies the free-diposal property $Y_{j}-D=Y_{j}$.

In this model the production sets do not need to be convex. Without convexity, the analysis strongly rests on a free-disposal assumption: we suppose that there exists a free-disposal cone $D$ common to all the firms. Actually, if the firms have different free-disposal cones $D_{j}$, we can consider a common cone $D=\cap_{j=1}^{n} D_{j}$. We only need to assume that the interior of the cone $D$ is nonempty if each cone $D_{j}$ satisfy the condition in Assumption $(\mathrm{P})$.

The properties of $D$ implies that there exists $e \in \operatorname{int} D$ such that $D \subset e^{+}=$ $\{0\} \cup\{x \in L \mid x \cdot e>0\}$. This reference commodity bundle $e$, which can be seen as a specie, will be assigned an important role throughout this paper. The usual setting is $L=\mathbb{R}^{\ell}, D=\mathbb{R}_{+}^{\ell}$ and $e=(1, \ldots, 1)$.

Remark 1 : Under Assumption (P), for every $j$, the boundary $\partial Y_{j}$ of the production set $Y_{j}$ is exactly the set of weakly efficient productions, that is $\partial Y_{j}=\left\{y_{j} \in Y_{j} \mid\left[\left\{y_{j}\right\}+\operatorname{int} D\right] \cap Y_{j}=\emptyset\right\}$. As it is well known, equilibria of 
an economy in which the production sets are not supposed convex may not be aggregate productive efficient [see the example in Beato and Mas-Colell (1985)]. However, in order to get equilibria individually weakly productive efficient, we shall consider productions in the boundaries $\partial Y_{j}$ of the production sets.

Note that if, for the price vector $p \in L, p \cdot e \leq 0$, then the firms could prefer, in terms of profits, a non efficient production $\tilde{y}_{j}=y_{j}-t e$, with $t>0$, to the efficient production $y_{j} \in \partial Y_{j}$. In this case, there is actually no incentive for the firms to be efficient in their production choice. To be consistent with our efficiency requirement, we shall consider price vectors in the price set $P=\left(-D^{\circ}\right) \backslash\{0\} \subset e+$, where $D^{\circ}$ denotes the negative polar cone of $D$. This condition is clearly satisfied in the classical framework with non negative prices when $L=\mathbb{R}^{\ell}, D=\mathbb{R}_{+}^{\ell}$ and $e=(1, \ldots, 1)$.

Let us finally point out that our setting makes room for negative prices. Indeed, if the commodity space is endowed with an order structure such that the positive cone $L_{+}$is a closed, convex, pointed cone of vertex 0 with a nonempty interior, and if the free-disposal assumption is satisfied with $L_{+}$, then it is satisfied for any closed, convex pointed cone $D$ of vertex 0 contained in $L_{+}$, and we then have $L_{+} \subset-D^{\circ}$. This means that the price set is larger than the set of non negative prices. The smaller is the free disposal cone $D$, the larger is the price set $P$.

We consider a finite number $m$ of consumers (indexed by $i=1, \ldots, m$ ). The consumption set $X_{i} \subset L$ is the subset of all possible consumptions for consumer $i$, given her physical constraints. The tastes of this consumer are described by a binary preference relation ${ }^{1} \preceq_{i}$ on $X_{i}$, and her wealth is given by a function $r_{i}: P \times \prod_{j=1}^{n} \partial Y_{j} \rightarrow \mathbb{R}$, i.e., given the price vector $p \in P$ and the weakly efficient productions $\left(y_{j}\right) \in \prod_{j=1}^{n} \partial Y_{j}$, the wealth of consumer $i$ is $r_{i}\left(p,\left(y_{j}\right)\right)$. This abstract wealth structure clearly encompasses the case of a private ownership economy, in which $r_{i}\left(p,\left(y_{j}\right)\right)=\sum_{j=1}^{n} \theta_{i j} p \cdot y_{j}+p \cdot \omega_{i}$, where the $\theta_{i j}$ denote the consumers' shares in the production processes and satisfy $\theta_{i j} \geq 0$ for all $i, j$ and $\sum_{i=1}^{m} \theta_{i j}=1$ for all $j$, and where the $\omega_{i}$ denote the consumers' initial individual endowments and satisfy $\sum_{i=1}^{m} \omega_{i}=\omega$.

\section{Assumption (C)}

For every $i, X_{i}$ is a non-empty, closed, convex subset of $L$ such that $\mathcal{A} X_{i} \subset e^{+}$, $\preceq_{i}$ is a continuous, convex and non-satiated complete preorder ${ }^{2}$ on $X_{i}$, and $r_{i}$

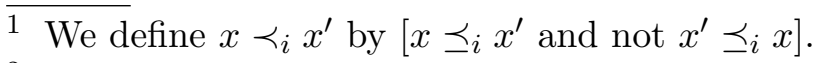

$2 \mathcal{A} X_{i}$ is the asymptotic cone to $X_{i}$, that is $\mathcal{A} X_{i}=\cap_{k \geq 0} \Gamma^{k}$, where $\Gamma^{k}$ denotes the smallest closed, convex cone with vertex 0 containing the set $\left\{x_{i} \in X_{i} \mid\left\|x_{i}\right\| \geq k\right\}$; for every $x_{i} \in X_{i}$, the subsets $\left\{x \in X_{i} \mid x \preceq_{i} x_{i}\right\}$ and $\left\{x \in X_{i} \mid x_{i} \preceq_{i} x\right\}$ are closed, the subset $\left\{x \in X_{i} \mid x_{i} \preceq_{i} x\right\}$ is convex, and, there exists $x \in X_{i}$ such that $x_{i} \prec_{i} x$; 
is a continuous function on $P \times \prod_{j=1}^{n} \partial Y_{j}$, satisfying $r_{i}\left(\lambda p,\left(y_{j}\right)\right)=\lambda r_{i}\left(p,\left(y_{j}\right)\right)$ for every $\lambda>0$ and every $\left(p,\left(y_{j}\right)\right) \in P \times \prod_{j=1}^{n} \partial Y_{j}$.

This assumption is classical, apart from the condition that $\mathcal{A} X_{i} \subset e^{+}$for every $i$. It is satisfied in the particular case where $L=\mathbb{R}^{\ell}, e=(1, \ldots, 1)$ and all the consumption set $X_{i}$ are bounded below. Nevertheless, our assumption allows us to consider consumption sets which are not bounded from below, like the following one in $\mathbb{R}^{2}$ :

$$
\left\{\left(x_{1}, x_{2}\right) \in \mathbb{R}^{2} \mid x_{1} \geq 0, x_{2} \geq-\ln \left(x_{1}+1\right)\right\} .
$$

This assumption is sufficient to guarantee the boundedness of the consumption allocations for given productions.

We let $X=\sum_{i=1}^{m} X_{i}+D-\omega$. In the following lemma, we gather some properties of $X$ which are direct consequences of Assumption (C). The proof is given in Appendix.

Lemma 1 Under Assumption (C), $X$ is a nonempty, closed, convex subset of $L$, different from $L$ and satisfying $X+D=X$. Furthermore, for all compact subset $K$ of $X$, the following set is compact:

$$
\left\{\left(\left(x_{i}\right), d\right) \in \prod_{i=1}^{m} X_{i} \times D \mid \sum_{i=1}^{m} x_{i}+d-\omega \in K\right\} .
$$

For every real number $t \geq 0$, we call $t$-attainable production allocation any element in:

$$
A_{t}=\left\{\left(y_{j}\right) \in \prod_{j=1}^{n} \partial Y_{j} \mid \sum_{j=1}^{n} y_{j}+t e \in X\right\} .
$$

$A_{t}$ is the set of all productions which become attainable when $t$ units of the reference commodity bundle $e$ are added to the total initial endowments $\omega$. $A_{t}$ is closed under the Assumptions (P) and (C) since $X$ is closed and the production sets are closed. $A_{0}$ clearly denotes the set of all attainable production allocations.

Remark 2 : Note that, from Lemma 1, if we let $Y_{0}=-X$, then under Assumptions (P) and (C), for every $j=0,1, \ldots, n$, the set $Y_{j}$ is a nonempty, closed subset of $L$, different from $L$ and satisfying the free-disposal property $Y_{j}-D=Y_{j}$. We can therefore apply the following lemma [see the proof in Appendix] which generalizes Lemma 5.1 in Bonnisseau and Cornet (1988) to our setting. We denoteby $\operatorname{proj}_{e^{\perp}}$ the orthogonal projection on $e^{\perp}$, the orthogonal space $^{3}$ to $e \in \operatorname{int} D$.

$\preceq_{i}$ is a complete, reflexive, transitive binary relation.

3 That is, $e^{\perp}=\{s \in L \mid s \cdot e=0\}$. 
Lemma 2 Let $Y_{j}$ be a non-empty, closed subset of $L$, different from $L$ and satisfying $Y_{j}-D=Y_{j}$. Then, for every $s \in e^{\perp}$, there is a unique real number, denoted by $\lambda_{j}(s)$, such that $s-\lambda_{j}(s) e \in \partial Y_{j}$. The function $\lambda_{j}: e^{\perp} \rightarrow \mathbb{R}$ is Lipschitz continuous, and the mapping $\Lambda_{j}: s \in e^{\perp} \mapsto s-\lambda_{j}(s) e \in \partial Y_{j}$ is an homeomorphism. The inverse of $\Lambda_{j}$ is the restriction of the orthogonal projection on $e^{\perp}$ to $\partial Y_{j}$. Finally, we have:

$$
Y_{j}=\left\{y_{j} \in L \mid \lambda_{j}\left(\operatorname{proj}_{e^{\perp}}\left(y_{j}\right)\right)+\frac{y_{j} \cdot e}{\|e\|^{2}} \leq 0\right\} .
$$

Consequently, under Assumptions (P) and (C), $\partial Y_{j}$ is homeomorphic to the Euclidean space $e^{\perp}$ for every $j=0,1, \ldots, n$. We define the mapping $\Lambda$ : $\left(e^{\perp}\right)^{n} \rightarrow \prod_{j=1}^{n} \partial Y_{j}$ by $\Lambda(s)=\left(\Lambda_{j}\left(s_{j}\right)\right)$ for $s=\left(s_{j}\right)$ and we remark that it is an homeomorphism. Finally, $Y_{j}$ is described by the transformation function $g_{j}\left(y_{j}\right)=\lambda_{j}\left(\operatorname{proj}_{e^{\perp}}\left(y_{j}\right)\right)+\frac{y_{j} \cdot e}{\|e\|^{2}}$.

For every real number $t \geq 0$, let $M_{t}$ be the inverse image under $\Lambda$ of $A_{t}$ :

$$
M_{t}:=\Lambda^{-1}\left(A_{t}\right)=\left\{s=\left(s_{j}\right) \in\left(e^{\perp}\right)^{n} \mid \sum_{j=1}^{n} \Lambda_{j}\left(s_{j}\right)+t e \in X\right\} .
$$

Let $\theta$ be the function defined on $\left(e^{\perp}\right)^{n}$ by:

$$
\theta(s)=\sum_{j=1}^{n} \lambda_{j}\left(s_{j}\right)+\lambda_{0}\left(-\sum_{j=1}^{n} s_{j}\right)
$$

Then $\theta$ is Lipschitz continuous under the Assumptions (P) and (C) and, for every real number $t \geq 0$, we have:

$$
M_{t}=\left\{s \in\left(e^{\perp}\right)^{n} \mid \theta(s) \leq t\right\},
$$

and

$$
A_{t}=\left\{\left(y_{j}\right) \in \prod_{j=1}^{n} \partial Y_{j} \mid \theta\left[\left(\operatorname{proj}_{e^{\perp}}\left(y_{j}\right)\right)\right] \leq t\right\} .
$$

The function $\theta$ measures the distance of the global production $\sum_{j=1}^{n} y_{j}$ to the global consumption set $X$. More precisely, if $\sum_{j=1}^{n} y_{j} \notin X$, then $\sum_{j=1}^{n} y_{j}+$ $\theta\left(\left(\operatorname{proj}_{e^{\perp}}\left(y_{j}\right)\right)\right) e \in \partial X$.

Under Assumptions (P) and (C), if the set $A_{t}$ is compact, then the continuity of the mapping $\Lambda^{-1}$ implies that the set $M_{t}$ is compact. Hence, the convex hull co $M_{t}$ of the set $M_{t}$ is compact. When $A_{t}$ is nonempty and compact, we let $\Theta(t)=\max \left\{\theta(s) \mid s \in \operatorname{co} M_{t}\right\}$.

We suppose that the firms in the economy follow a general pricing rule $\varphi$, which is a correspondence from $P \times \prod_{j=1}^{n} \partial Y_{j}$ into $\left(e^{+}\right)^{n}$. This means that, given the price vector $p \in P$ and the weakly efficient productions $\left(y_{j}\right) \in \prod_{j=1}^{n} \partial Y_{j}$, each 
firm $j$ chooses a price vector $q_{j} \in e^{+}$so that $\left(q_{j}\right) \in \varphi\left(p,\left(y_{j}\right)\right)$. Note that, in the classical framework, $D=\mathbb{R}_{+}^{\ell}, P=\mathbb{R}_{+}^{\ell} \backslash\{0\}$ and $\varphi\left(p,\left(y_{j}\right)\right) \subset\left(\mathbb{R}_{+}^{\ell}\right)^{n}$ for every $\left(p,\left(y_{j}\right)\right) \in \mathbb{R}_{+}^{\ell} \backslash\{0\} \times \prod_{j=1}^{n} \partial Y_{j}$. The possibility of prices $q_{j}$ in $e^{+}$, i.e., of price vectors not necessarily belonging to the price space $P$, is purely a technical requirement. We shall use it in Section 4 to deduce the existence of a marginal pricing equilibrium from the existence of an equilibrium for general pricing rules with bounded losses.

In the following, we will consider without any loss of generality normalized price vector. Indeed, the wealth functions $r_{i}$ are supposed to be homogeneous with respect to the price vector and we will assume the same property for the pricing rule. Let $\mathcal{H}=\{p \in L \mid p \cdot e=1\} \subset e^{+}$and $S=P \cap \mathcal{H}$. The choice of $e$ implies that $S$ is compact. The normalized general pricing rule, denoted by $\bar{\varphi}$, is defined for every $\left(p,\left(y_{j}\right)\right) \in S \times \prod_{j=1}^{n} \partial Y_{j}$ by:

$$
\bar{\varphi}\left(p,\left(y_{j}\right)\right)=\varphi\left(p,\left(y_{j}\right)\right) \cap \mathcal{H}^{n} .
$$

\section{Assumption (PR)}

For every $\left(p,\left(y_{j}\right)\right) \in P \times \prod_{j=1}^{n} \partial Y_{j}, \varphi\left(p,\left(y_{j}\right)\right)$ is a nonempty, closed, convex cone of vertex 0 , different from $\{0\}$, included in $\left(e^{+}\right)^{n}$, and $\varphi\left(\lambda p,\left(y_{j}\right)\right)=$ $\varphi\left(p,\left(y_{j}\right)\right)$ for every $\lambda>0 . \bar{\varphi}\left(p,\left(y_{j}\right)\right) \subset S^{n}$ for every $\left(p,\left(y_{j}\right)\right) \in S \times A_{0}$, and $\bar{\varphi}$ is an upper hemi-continuous correspondence on $S \times \prod_{j=1}^{n} \partial Y_{j}$ with nonempty, convex, compact values in $\mathcal{H}^{n}$.

The production sector is said to be at equilibrium for the price vector $p$ and the production allocation $\left(y_{j}\right)$ if each firm can actually choose the price vector $p$, that is if $(p, \ldots, p) \in \varphi\left(p,\left(y_{j}\right)\right)$. We denote by $P E$ the set of normalized production equilibria, that is:

$$
P E=\left\{\left(p,\left(y_{j}\right)\right) \in S \times \prod_{j=1}^{n} \partial Y_{j} \mid(p, \ldots, p) \in \bar{\varphi}\left(p,\left(y_{j}\right)\right)\right\} .
$$

The set of the normalized attainable production equilibria is then:

$$
A P E=\left\{\left(p,\left(y_{j}\right)\right) \in P E \mid\left(y_{j}\right) \in A_{0}\right\} .
$$

Note that, under Assumptions (P), (C) and (PR), APE is closed since the normalized pricing rule $\bar{\varphi}$ is upper hemi-continuous and the set $A_{0}$ is closed.

\section{Existence of equilibria for general pricing rules}

Let us first present the notion of equilibrium in an economy with a general pricing rule. 
Definition 1 An element $\left(\left(x_{i}^{*}\right),\left(y_{j}^{*}\right), p^{*}\right)$ in $L^{m} \times L^{n} \times P$ is an equilibrium of the economy $\mathcal{E}=\left(L,\left(X_{i}, \preceq_{i}, r_{i}\right),\left(Y_{j}\right), \varphi, \omega\right)$ if:

(a) for every $i, x_{i}^{*}$ is a greater element for $\preceq_{i}$ in $B_{i}\left(p^{*},\left(y_{j}^{*}\right)\right)=\left\{x_{i} \in X_{i} \mid\right.$ $\left.p^{*} \cdot x_{i} \leq r_{i}\left(p^{*},\left(y_{j}^{*}\right)\right)\right\}$

(b) for every $j, y_{j}^{*} \in \partial Y_{j}$ and $\left(p^{*}, \ldots, p^{*}\right) \in \varphi\left(p^{*},\left(y_{j}^{*}\right)\right)$;

(c) $\sum_{i=1}^{m} x_{i}^{*}=\sum_{j=1}^{n} y_{j}^{*}+\omega$.

This definition is a generalization of the notion of a Walras equilibrium to our setting. The firms' profit maximization condition is replaced by the condition that they follow the general pricing rule $\varphi$.

To establish the existence of equilibria in the economy $\mathcal{E}$, two further assumptions on the wealth's distribution are needed. The first one is a condition on the consumers' individual wealth:

\section{Assumption (R)}

For every $i$ and every $\left(p,\left(y_{j}\right)\right) \in A P E, r_{i}\left(p,\left(y_{j}\right)\right)>\inf p \cdot X_{i}$. Furthermore, $\sum_{i=1}^{m} r_{i}\left(p,\left(y_{j}\right)\right)=p \cdot\left(\sum_{j=1}^{n} y_{j}+\omega\right)$ for every $\left(p,\left(y_{j}\right)\right) \in P E$.

The second condition gathers three independent assumptions made in previous works, namely, the boundedness, the survival and the bounded losses assumptions. To limit these assumptions to a bounded subset of production allocations, we shall posit the following. Actually, this constitutes the major improvement with respect to the previous existence results. First, we only need to have a bounded attainable allocation set only with a limited increase of the initial endowments whereas it is usually assumed that the attainable allocations are bounded for all greater initial endowments. We also posit the survival assumption only on a bounded neighborhood of the attainable allocations and not for all production equilibria. Finally, the bounded losses assumption is also restricted to productions which are at a finite distance of the attainable productions, in the sense that they becomes attainable with a bounded increase of the initial endowments.

\section{Assumption (BLS)}

There exists a real number $t_{0} \geq 0$ such that $A_{t_{0}}$ is nonempty and bounded, and:

(BS) for every $t \in\left[0, t_{0}\left[\right.\right.$ and every $\left(p,\left(y_{j}\right)\right) \in P E$, if $\sum_{j=1}^{n} y_{j}+$ te $\in X$, then $p \cdot\left(\sum_{j=1}^{n} y_{j}+t e\right)>\inf p \cdot X$;

(BL) for every $t \in\left[t_{0}, \Theta\left(t_{0}\right)\right]$, every $\left(p,\left(y_{j}\right)\right) \in S \times A_{t}$ and every $\left(q_{j}\right) \in$ $\bar{\varphi}\left(p,\left(y_{j}\right)\right)$, if $p \in-N_{X}\left(\sum_{j=1}^{n} y_{j}+t e\right)$, then there exists $\left(\hat{y}_{j}\right) \in A_{t_{0}}$ such that $\sum_{j=1}^{n}\left(q_{j}-p\right) \cdot\left(y_{j}-\hat{y}_{j}\right)>0$.

Remark 3 : Remark that Assumption (BLS) implies that: 
For every $t \in\left[0, \Theta\left(t_{0}\right)\right]$ and every $\left(p,\left(y_{j}\right)\right) \in P E$, if $\sum_{j=1}^{n} y_{j}+$ te $\in X$, then $p \cdot\left(\sum_{j=1}^{n} y_{j}+t e\right)>\inf p \cdot X$.

Indeed, it is clear for every $t \in\left[0, t_{0}[\right.$ part (BS) of Assumption (BLS). For every $t \in\left[t_{0}, \Theta\left(t_{0}\right)\right]$, if $\left(p,\left(y_{j}\right)\right) \in P E$ and $\sum_{j=1}^{n} y_{j}+t e \in \partial X$, then $(p, \ldots, p) \in$ $\bar{\varphi}\left(p,\left(y_{j}\right)\right)$ and $\sum_{j=1}^{n}(p-p) \cdot\left(y_{j}-\hat{y}_{j}\right)=0$ for any $\left(\hat{y}_{j}\right) \in \prod_{j=1}^{n} \partial Y_{j}$. Consequently, the contraposition of Part (BL) of Assumption (BLS) implies that $p \notin-N_{X}\left(\sum_{j=1}^{n} y_{j}+t e\right)$.

This means that, at production equilibria, if $t$ units of the reference commodity bundle $e$ are added to the total initial endowments $\omega$ in such a way that the production allocation becomes attainable, then the total wealth of the economy is above the consumers' subsistence level.

Remark 4 : Part (BL) of Assumption (BLS) is a weakening of the following bounded losses assumption, made in Bonnisseau and Cornet (1988), where the pricing rule is supposed to take its values in $P^{n}$. It means that the normalized general pricing rule $\bar{\varphi}$ cannot generate losses lower than $\alpha$. Note that the existence theorem in Bonnisseau and Cornet (1988) requires a stronger boundedness assumption, which is, with our notations, $A_{t}$ is bounded for every $t \geq 0$.

Assumption (BL) There exists a real number $\alpha$ such that, for every $\left(p,\left(y_{j}\right)\right) \in$ $S \times \prod_{j=1}^{n} \partial Y_{j}$ and every $\left(q_{j}\right) \in \bar{\varphi}\left(p,\left(y_{j}\right)\right), \sum_{j=1}^{n} q_{j} \cdot y_{j} \geq \alpha$.

Indeed, let $\left(\hat{y}_{j}\right) \in \prod_{j=1}^{n} \partial Y_{j}$ and $\tau=\theta(\hat{s})$, where $\hat{s}=\left(\operatorname{proj}_{e^{\perp}}\left(\hat{y}_{j}\right)\right)$. Let $t_{0} \geq \tau$ and $t_{0}>\sum_{j=1}^{n} \sup \left\{\sigma \cdot \hat{y}_{j} \mid \sigma \in S-S\right\}+\sup \{q \cdot x \mid q \in S\}-\alpha$, where $x$ is any fixed element in $X$. For every $t \geq t_{0}$, every $\left(p,\left(y_{j}\right)\right) \in S \times A_{t}$ and every $\left(q_{j}\right) \in \bar{\varphi}\left(p,\left(y_{j}\right)\right)$, if $p \in-N_{X}\left(\sum_{j=1}^{n} y_{j}+t e\right)$, then $p \cdot\left(\sum_{j=1}^{n} y_{j}+t e\right)=$ $p \cdot \sum_{j=1}^{n} y_{j}+t=\inf p \cdot X$. If $\varphi$ satisfies Assumption (BL), then $\sum_{j=1}^{n} q_{j} \cdot y_{j} \geq \alpha$ and:

$$
\begin{aligned}
\sum_{j=1}^{n} q_{j} \cdot y_{j} & >\sum_{j=1}^{n} \sup \left\{\sigma \cdot \hat{y}_{j} \mid \sigma \in S-S\right\}+\sup \{q \cdot x \mid q \in S\}-t_{0} \\
& \geq \sum_{j=1}^{n}\left(q_{j}-p\right) \cdot \hat{y}_{j}+\inf p \cdot X-t \\
& \geq \sum_{j=1}^{n}\left(q_{j}-p\right) \cdot \hat{y}_{j}+p \cdot \sum_{j=1}^{n} y_{j},
\end{aligned}
$$

hence $\varphi$ satisfies Part (BL) of Assumption (BLS).

Remark 5 : An interesting feature of Assumption (BLS) is that it allows to get an unified proof for loss free and bounded losses pricing rules. Indeed, in Bonnisseau and Cornet (1988), the proofs are different essentially because, in the case of loss free pricing rules, the boundedness assumption is only $A_{0}$ is bounded. Actually, under this condition, Assumption (BLS) (with $t_{0}=0$ ) is 
satisfied for loss free pricing rule, that is when $\alpha=0$ in Assumption (BL), under a strong survival assumption like $\omega \in \operatorname{int}\left(\sum_{i=1}^{m} X_{i}+D\right)$. Indeed, let $\hat{y}_{j}=0-\lambda_{j}(0) e$ for every $j$, and $\left(q_{j}\right) \in \bar{\varphi}\left(p,\left(\hat{y}_{j}\right)\right)$ for any $p \in S$. One has $\sum_{j=1}^{n} q_{j} \cdot \hat{y}_{j}=-\left(\sum_{j=1}^{n} \lambda_{j}(0)\right) \geq 0$, hence $\sum_{j=1}^{n} \hat{y}_{j} \in D$. From $\omega \in \operatorname{int}\left(\sum_{j=1}^{n} X_{i}+\right.$ $D)$ we get $0 \in \operatorname{int} X$. Since $X+D=X, \sum_{j=1}^{n} \hat{y}_{j} \in D \subset X$, i.e., $\left(\hat{y}_{j}\right) \in A_{0}$. Note also that $\inf p \cdot X<0$ for all $p \in S$ since $0 \in \operatorname{int} X$. Now, let $t \geq 0$, $\left(p,\left(y_{j}\right)\right) \in S \times A_{t}$ and $\left(q_{j}\right) \in \bar{\varphi}\left(p,\left(y_{j}\right)\right)$. If $p \in-N_{X}\left(\sum_{j=1}^{n} y_{j}+t e\right)$, then, since $\bar{\varphi}$ is a loss-free pricing rule,

$$
\sum_{j=1}^{n}\left(q_{j}-p\right) \cdot\left(y_{j}-\hat{y}_{j}\right)=\sum_{j=1}^{n}\left(q_{j}-p\right) \cdot y_{j} \geq 0-\inf p \cdot X+t>0 .
$$

Consequently, Assumption (BLS) is satisfied since, when $t_{0}=0$, part (BS) is obviously satisfied.

The classical competitive framework $\grave{a}$ la Arrow-Debreu, where the production sets are convex and the firms are supposed to maximize their profits, corresponds to the pricing rule given by $\varphi\left(p,\left(y_{j}\right)\right)=\prod_{j=1}^{n} N_{Y_{j}}\left(y_{j}\right)$, where $N_{Y_{j}}\left(y_{j}\right)$ denotes the normal cone to the closed, convex set $Y_{j}$ at $y_{j} \in Y_{j}$. In this setting, if $A_{0}$ is supposed compact, then Assumption (BLS) (with $t_{0}=0$ ) is a consequence of the aggregate survival assumption $\omega \in \operatorname{int}\left(\sum_{i=1}^{m} X_{i}+D\right)$ and the possibility of inaction, that is $0 \in Y_{j}$ for all $j$. Indeed, this is a consequence of the previous argument. The pricing rule is loss free since, for every $j$, every $y_{j} \in \partial Y_{j}$ and every $q_{j} \in N_{Y_{j}}\left(y_{j}\right), q_{j} \cdot y_{j} \geq q_{j} \cdot 0=0$.

When the production sets are convex, if $A_{0}$ is nonempty and bounded, then $A_{t}$ is also nonempty and bounded for all $t \geq 0$. This is no more true in the non-convex case.

We shall in this section establish the following existence result.

Theorem 1 The economy $\mathcal{E}=\left(L,\left(X_{i}, \preceq_{i}, r_{i}\right),\left(Y_{j}\right), \varphi, \omega\right)$ has an equilibrium under Assumptions (P), (C), (PR), (R) and (BLS).

\subsection{The preliminary result}

In order to prove the existence of equilibria in the economy $\mathcal{E}$, we shall follow Debreu, Gale and Nikaido's approach. We first give a result on an arbitrary excess demand correspondence, from which we deduce the existence of equilibria for general pricing rules in an economy under increasing returns.

Let thus $z$ be an arbitrary excess demand correspondence. As in Bonnisseau and Cornet (1991), we posit the following assumption: 
Assumption (ED)

$z$ is an upper hemi-continuous correspondence on $S \times \prod_{j=1}^{n} \partial Y_{j}$ with nonempty, convex, compact values in L. Furthermore, for every $\left(p,\left(y_{j}\right)\right) \in A P E$, $z\left(p,\left(y_{j}\right)\right) \in T_{X}\left(\sum_{j=1}^{n} y_{j}\right)$ and $\sup p \cdot z\left(p,\left(y_{j}\right)\right) \leq 0$.

Remark 6 : The condition that $\sup p \cdot z\left(p,\left(y_{j}\right)\right) \leq 0$ for every $\left(p,\left(y_{j}\right)\right) \in$ $A P E$ is the translation of the Walras law in our model, as a consequence of the fact that the total wealth of the economy is shared among the consumer [Assumption (R)].

We can now establish our preliminary result on the excess demand correspondence $z$.

Theorem 2 Under Assumptions (P), (C), (PR), (BLS) and (ED), there is an element $\left(p^{*},\left(y_{j}^{*}\right)\right)$ in $S \times \prod_{j=1}^{n} \partial Y_{j}$ satisfying:

(a) $\sum_{j=1}^{n} y_{j}^{*} \in X$;

(b) $\left(p^{*}, \ldots, p^{*}\right) \in \bar{\varphi}\left(p^{*},\left(y_{j}^{*}\right)\right)$;

(c) $z\left(p^{*},\left(y_{j}^{*}\right)\right) \cap-\mathbb{R}_{+} e \neq \emptyset$.

The proof of Theorem 1 is divided into 3 steps.

\subsubsection{Step 1}

In the first step, we construct auxiliary excess demand correspondences. Let $\alpha>0$ be a real number, and let $S_{\alpha}=S+\alpha \bar{B}_{e^{\perp}}(0,1)$, where $\bar{B}_{e^{\perp}}(0,1)$ denotes the closed unit ball in $e^{\perp}$. In the following, we denote by $\operatorname{proj}_{e^{\perp}}$ the orthogonal projection onto $e^{\perp}$, by $\pi$ the projection mapping from $L$ to $S$, and we define the mapping $\operatorname{proj}_{X}$, from $L$ to $X$, by:

$$
\operatorname{proj}_{X}(y)=\operatorname{proj}_{e^{\perp}}(y)+\max \left\{\lambda_{0}\left(-\operatorname{proj}_{e^{\perp}}(y)\right), \frac{y \cdot e}{\|e\|^{2}}\right\} e .
$$

Recall that, under Assumptions (P) and (C), the set $A_{t_{0}}$ is closed. Moreover, from Assumption (BLS), $A_{t_{0}}$ is nonempty and bounded. Therefore, $A_{t_{0}}$ and $M_{t_{0}}$ are nonempty and compact. In this step, we shall work in the nonempty, convex, compact set $\mathcal{B}_{\gamma}=\operatorname{co} M_{t_{0}}+\gamma \bar{B}_{\left(e^{\perp}\right)^{n}}(0,1)$. Since $\mathcal{B}_{\gamma}$ is not necessarily included in $M_{\Theta\left(t_{0}\right)}$, there may be elements $\left(y_{j}\right) \in \Lambda\left(\mathcal{B}_{\gamma}\right)$ such that $\sum_{j=1}^{n} y_{j}+t e \in$ $\partial X$ with $t>\Theta\left(t_{0}\right)$. The following lemma, proved in the Appendix, establish that if Assumption (BLS) is satisfied, then there exists $\gamma>0$ small enough such that the elements $\left(y_{j}\right) \in \mathcal{B}_{\gamma}$ also satisfy the conclusions of Part (BL) of Assumption (BLS) for $t>\Theta\left(t_{0}\right)$.

Lemma 3 Under Assumption (BLS), there exists $\gamma>0$ such that: 
(BS) for every $\left(p,\left(y_{j}\right)\right) \in S \times \Lambda\left(\mathcal{B}_{\gamma}\right)$, if $\left(p,\left(y_{j}\right)\right) \in P E$ and $\sum_{j=1}^{n} y_{j}+$ te $\in X$ with $t \geq 0$, then $p \cdot\left(\sum_{j=1}^{n} y_{j}+\right.$ te $)>\inf p \cdot X$;

(BL) for every $\left(p,\left(y_{j}\right)\right) \in S \times \Lambda\left(\mathcal{B}_{\gamma}\right)$ and every $\left(q_{j}\right) \in \bar{\varphi}\left(p,\left(y_{j}\right)\right)$, if $p \in$ $-N_{X}\left(\sum_{j=1}^{n} y_{j}+t e\right)$ with $t \geq t_{0}$, then there exists $\left(\hat{y}_{j}\right) \in A_{t_{0}}$ such that $\sum_{j=1}^{n}\left(q_{j}-p\right) \cdot\left(y_{j}-\hat{y}_{j}\right)>0$.

Let now $U$ be the set defined as follows:

$$
U=\left\{\left(p,\left(y_{j}\right)\right) \in S_{\alpha} \times \Lambda\left(\mathcal{B}_{\gamma}\right) \mid p \notin-N_{X}\left(\operatorname{proj}_{X}\left(\sum_{j=1}^{n} y_{j}\right)\right)\right\} .
$$

From the properties of the normal cone and the continuity of the mapping $\operatorname{proj}_{X}, U$ is an open subset of $S_{\alpha} \times \Lambda\left(\mathcal{B}_{\gamma}\right)$ (for its relative topology) which contains $A P E$ from Lemma 3. Moreover, from Assumption (BLS), there exists $t_{0} \geq 0$ such that $A_{t_{0}}$ is bounded, hence $A_{0}$ is bounded and $A P E$ is compact from Assumptions (P), (C) and (PR). Consequently, there is a real number $\varepsilon_{0}>0$ such that, for every $\left.\varepsilon \in\right] 0, \varepsilon_{0}\left[\right.$, the closed ball $\bar{B}(A P E, \varepsilon)$ in $S_{\alpha} \times \Lambda\left(\mathcal{B}_{\gamma}\right)$ is a subset of $U$, and, for every $\left(p,\left(y_{j}\right)\right) \in \bar{B}(A P E, \varepsilon), p$ belongs to the relative interior of $S_{\alpha}$. Hence, for every $\left.\varepsilon \in\right] 0, \varepsilon_{0}[$, there is a continuous function $\tau^{\varepsilon}: S_{\alpha} \times \Lambda\left(\mathcal{B}_{t_{0}}\right) \rightarrow[0,1]$ satisfying:

$$
\begin{aligned}
& \tau^{\varepsilon}\left(p,\left(y_{j}\right)\right)=1 \text { if }\left(p,\left(y_{j}\right)\right) \in A P E, \\
& \tau^{\varepsilon}\left(p,\left(y_{j}\right)\right)=0 \text { if }\left(p,\left(y_{j}\right)\right) \notin \bar{B}(A P E, \varepsilon) .
\end{aligned}
$$

We now define the correspondence $\Psi$, from $S_{\alpha} \times \Lambda\left(\mathcal{B}_{\gamma}\right)$ into $L$, as follows:

$$
\Psi\left(p,\left(y_{j}\right)\right)=\operatorname{int} T_{X}\left(\operatorname{proj}_{X}\left(\sum_{j=1}^{n} y_{j}\right)\right) \cap\{u \in L \mid p \cdot u<0\} \cap B_{L}(0,1),
$$

where $B_{L}(0,1)$ denotes the open unit ball in $L . \Psi$ has an open graph and convex values, and $\Psi\left(p,\left(y_{j}\right)\right) \neq \emptyset$ for every $\left(p,\left(y_{j}\right)\right) \in U$. Let thus $\psi: U \rightarrow L$ be a continuous selection ${ }^{4}$ of $\Psi$, and $\zeta$ be the correspondence from $S_{\alpha} \times \Lambda\left(\mathcal{B}_{\gamma}\right)$ into $L$ defined by:

$$
\zeta\left(p,\left(y_{j}\right)\right)= \begin{cases}\left\{\psi\left(p,\left(y_{j}\right)\right)\right\} & \text { if }\left(p,\left(y_{j}\right)\right) \in U \\ \bar{B}_{L}(0,1) & \text { otherwise }\end{cases}
$$

$\zeta$ is clearly an upper hemi-continuous correspondence with nonempty, convex, compact values.

\footnotetext{
$\overline{4}$ For every $\left(p,\left(y_{j}\right)\right) \in U, \psi\left(p,\left(y_{j}\right)\right) \in \Psi\left(p,\left(y_{j}\right)\right)$. The existence of a selection is a consequence of a weak version of Michael's Theorem since $\Psi$ has an open graph.
} 
We finally define the auxiliary excess demand correspondence $z^{\varepsilon}$, for every $\varepsilon \in] 0, \varepsilon_{0}[$, as follows:

$$
z^{\varepsilon}\left(p,\left(y_{j}\right)\right)=\tau^{\varepsilon}\left(p,\left(y_{j}\right)\right) z\left(\pi(p),\left(y_{j}\right)\right)+\left(1-\tau^{\varepsilon}\left(p,\left(y_{j}\right)\right)\right) \zeta\left(p,\left(y_{j}\right)\right) .
$$

For every $\varepsilon \in] 0, \varepsilon_{0}\left[\right.$, $z^{\varepsilon}$ is an upper hemi-continuous correspondence with nonempty, convex, compact values satisfying the following properties:

$$
\begin{array}{ll}
z^{\varepsilon}\left(p,\left(y_{j}\right)\right)=z\left(p,\left(y_{j}\right)\right) & \text { if }\left(p,\left(y_{j}\right)\right) \in A P E, \\
z^{\varepsilon}\left(p,\left(y_{j}\right)\right)=\left\{\psi\left(p,\left(y_{j}\right)\right)\right\} & \text { if }\left(p,\left(y_{j}\right)\right) \in U \backslash \bar{B}(A P E, \varepsilon) .
\end{array}
$$

\subsubsection{Step 2}

We claim that, for every $\varepsilon \in] 0, \varepsilon_{0}\left[\right.$, there exists $\left(p^{\varepsilon},\left(y_{j}^{\varepsilon}\right)\right) \in \bar{B}(A P E, \varepsilon)$ and $x^{\varepsilon} \in$ $z^{\varepsilon}\left(p^{\varepsilon},\left(y_{j}^{\varepsilon}\right)\right)$ such that $x^{\varepsilon} \in \mathbb{R} e$. Furthermore, if $\sum_{j=1}^{n} y_{j}^{\varepsilon} \in X$, then $\left(p^{\varepsilon},\left(y_{j}^{\varepsilon}\right)\right) \in$ $A P E$.

First of all, let us point out that, for every $\varepsilon \in] 0, \varepsilon_{0}\left[\right.$, the properties of $z^{\varepsilon}$ imply that there exists a nonempty, convex, compact subset $K^{\varepsilon} \subset L$ such that $z^{\varepsilon}(p, \Lambda(s)) \subset K^{\varepsilon}$ for every $(p, s) \in S_{\alpha} \times \mathcal{B}_{\gamma}$. Moreover, from the upper hemi-continuity of the normalized pricing rule $\bar{\varphi}$ and the continuity of the projection mapping $\pi$, there exists a compact subset $\bar{S}$ in $\mathcal{H}$, containing $S$, such that $\bar{\varphi}(\pi(p), \Lambda(s)) \subset \bar{S}^{n}$ for every $(p, s) \in S_{\alpha} \times \mathcal{B}_{\gamma}$.

We now let $F^{\varepsilon}$ be the correspondence from $K^{\varepsilon} \times \mathcal{B}_{\gamma} \times S_{\alpha} \times \bar{S}^{n}$ into itself defined by:

$$
F^{\varepsilon}\left(x, s, p,\left(p_{j}\right)\right)=\prod_{\nu=1}^{4} F_{\nu}^{\varepsilon}\left(x, s, p,\left(p_{j}\right)\right)
$$

where:

$$
\begin{aligned}
& F_{1}^{\varepsilon}\left(x, s, p,\left(p_{j}\right)\right)=z^{\varepsilon}(p, \Lambda(s)), \\
& F_{2}^{\varepsilon}\left(x, s, p,\left(p_{j}\right)\right)=\left\{\left(\sigma_{j}\right) \in \mathcal{B}_{\gamma} \mid \forall\left(\sigma_{j}^{\prime}\right) \in \mathcal{B}_{\gamma}: \sum_{j=1}^{n}\left(p-p_{j}\right) \cdot\left(\sigma_{j}-\sigma_{j}^{\prime}\right) \geq 0\right\} \text {, } \\
& F_{3}^{\varepsilon}\left(x, s, p,\left(p_{j}\right)\right)=\left\{q \in S_{\alpha} \mid \forall q^{\prime} \in S_{\alpha}:\left(q-q^{\prime}\right) \cdot x \geq 0\right\} \text {, } \\
& F_{4}^{\varepsilon}\left(x, s, p,\left(p_{j}\right)\right)=\bar{\varphi}(\pi(p), \Lambda(s)) \text {. }
\end{aligned}
$$

From Assumption (PR), from the properties of $z^{\varepsilon}$ and from the maximum Theorem in Berge (1959), $F^{\varepsilon}$ is an upper hemi-continuous correspondence with non-empty, convex, compact values. Consequently, from Kakutani's theorem, for every $\varepsilon \in] 0, \varepsilon_{0}\left[\right.$, there exists a fixed point $\left(x^{\varepsilon}, s^{\varepsilon}, p^{\varepsilon},\left(p_{j}^{\varepsilon}\right)\right)$ of $F^{\varepsilon}$ satisfying $x^{\varepsilon} \in z^{\varepsilon}\left(p, \Lambda\left(s^{\varepsilon}\right)\right)$ and: 


$$
\begin{array}{r}
\sum_{j=1}^{n}\left(p^{\varepsilon}-p_{j}^{\varepsilon}\right) \cdot s_{j}^{\varepsilon} \geq \sum_{j=1}^{n}\left(p^{\varepsilon}-p_{j}^{\varepsilon}\right) \cdot s_{j} \text { for every }\left(s_{j}\right) \in \mathcal{B}_{\gamma} \\
p^{\varepsilon} \cdot x^{\varepsilon} \geq p \cdot x^{\varepsilon} \text { for every } p \in S_{\alpha} \\
\left(p_{j}^{\varepsilon}\right) \in \bar{\varphi}\left(\pi\left(p^{\varepsilon}\right), \Lambda\left(s^{\varepsilon}\right)\right)
\end{array}
$$

We let $\left(y_{j}^{\varepsilon}\right)=\Lambda\left(s^{\varepsilon}\right)$. The following claims establish that $\left(p^{\varepsilon},\left(y_{j}^{\varepsilon}\right)\right) \in \bar{B}(A P E, \varepsilon)$, $x^{\varepsilon} \in z^{\varepsilon}\left(p^{\varepsilon},\left(y_{j}^{\varepsilon}\right)\right) \cap \mathbb{R} e$, and $\left(p^{\varepsilon},\left(y_{j}^{\varepsilon}\right)\right) \in A P E$ if $\sum_{j=1}^{n} y_{j}^{\varepsilon} \in X$.

Claim $1\left(p^{\varepsilon},\left(y_{j}^{\varepsilon}\right)\right) \in U$.

Proof: Suppose, on the contrary, that $\left(p^{\varepsilon},\left(y_{j}^{\varepsilon}\right)\right) \notin U$. Since $\left(p^{\varepsilon},\left(y_{j}^{\varepsilon}\right)\right) \in S_{\alpha} \times$ $\Lambda\left(\mathcal{B}_{\gamma}\right)$, we have $p^{\varepsilon} \in-N_{X}\left(\operatorname{proj}_{X}\left(\sum_{j=1}^{n} y_{j}^{\varepsilon}\right)\right) \subset P$, hence $p^{\varepsilon} \in S$. Consequently, $p^{\varepsilon} \neq 0$ and $\operatorname{proj}_{X}\left(\sum_{j=1}^{n} y_{j}^{\varepsilon}\right) \in \partial X$, hence $\sum_{j=1}^{n} y_{j}^{\varepsilon}+t e \in \partial X$ with $t \geq 0$. We then deduce from Lemma 3 that $\left(p^{\varepsilon},\left(y_{j}^{\varepsilon}\right)\right) \notin P E$, that is $\left(p^{\varepsilon}, \ldots, p^{\varepsilon}\right) \notin \bar{\varphi}\left(p^{\varepsilon},\left(y_{j}^{\varepsilon}\right)\right)$. From (3) we get $\left(p^{\varepsilon}-p_{1}^{\varepsilon}, \ldots, p^{\varepsilon}-p_{n}^{\varepsilon}\right) \neq 0$, and (1) then implies that $s^{\varepsilon} \in \partial \mathcal{B}_{\gamma}$. Hence $\operatorname{proj}_{X}\left(\sum_{j=1}^{n} y_{j}^{\varepsilon}\right)=\sum_{j=1}^{n} y_{j}^{\varepsilon}+$ te with $t \geq t_{0}$.

We now remark that, since $\left(\hat{y}_{j}\right) \in A_{t_{0}}, \hat{s}=\left(\operatorname{proj}_{e^{\perp}}\left(\hat{y}_{j}\right)\right) \in M_{t_{0}} \subset \operatorname{int} \mathcal{B}_{\gamma}$, hence for $\rho>0$ small enough, $s=\hat{s}-\rho\left(p_{1}^{\varepsilon}-p^{\varepsilon}, \ldots, p_{n}^{\varepsilon}-p^{\varepsilon}\right) \in \mathcal{B}_{\gamma}$. Consequently, from (1), we get:

$$
\begin{aligned}
\sum_{j=1}^{n} p_{j}^{\varepsilon} \cdot y_{j}^{\varepsilon} & =\sum_{j=1}^{n} p^{\varepsilon} \cdot y_{j}^{\varepsilon}+\sum_{j=1}^{n}\left(p_{j}^{\varepsilon}-p^{\varepsilon}\right) \cdot s_{j}^{\varepsilon} \\
& \leq \sum_{j=1}^{n} p^{\varepsilon} \cdot y_{j}^{\varepsilon}+\sum_{j=1}^{n}\left(p_{j}^{\varepsilon}-p^{\varepsilon}\right) \cdot \hat{s}_{j}-\rho \sum_{j=1}^{n}\left(p_{j}^{\varepsilon}-p^{\varepsilon}\right) \cdot\left(p_{j}^{\varepsilon}-p^{\varepsilon}\right) \\
& <\sum_{j=1}^{n} p^{\varepsilon} \cdot y_{j}^{\varepsilon}+\sum_{j=1}^{n}\left(p_{j}^{\varepsilon}-p^{\varepsilon}\right) \cdot \hat{s}_{j} \\
& <\sum_{j=1}^{n} p^{\varepsilon} \cdot y_{j}^{\varepsilon}+\sum_{j=1}^{n}\left(p_{j}^{\varepsilon}-p^{\varepsilon}\right) \cdot \hat{y}_{j}
\end{aligned}
$$

But, since $p^{\varepsilon} \in-N_{X}\left(\operatorname{proj}_{X}\left(\sum_{j=1}^{n} y_{j}^{\varepsilon}\right)\right)$ and $\left(p_{j}^{\varepsilon}\right) \in \bar{\varphi}\left(p^{\varepsilon},\left(y_{j}^{\varepsilon}\right)\right)$ from (3), this contradicts Part (BL) of Lemma 3.

Claim $2\left(p^{\varepsilon},\left(y_{j}^{\varepsilon}\right)\right) \in \bar{B}(A P E, \varepsilon)$ and $\left(p^{\varepsilon},\left(y_{j}^{\varepsilon}\right)\right) \in A P E$ if $\sum_{j=1}^{n} y_{j}^{\varepsilon} \in X$.

Proof: Suppose first that $\sum_{j=1}^{n} y_{j}^{\varepsilon} \in X$, i.e. that $\left(y_{j}^{\varepsilon}\right) \in A_{0}$. Then $s^{\varepsilon} \in$ $M_{0} \subset \operatorname{int} \mathcal{B}_{\gamma}$, and (1) implies that $p^{\varepsilon}=p_{j}^{\varepsilon}$ for every $j$, hence $p^{\varepsilon} \in S$ since $\bar{\varphi}\left(\pi(p),\left(y_{j}^{\varepsilon}\right)\right) \subset S^{n}$ from Assumption (PR). From (3) we get $\left(p^{\varepsilon}, \ldots, p^{\varepsilon}\right) \in$ $\bar{\varphi}\left(p^{\varepsilon},\left(y_{j}^{\varepsilon}\right)\right)$, which means that $\left(p^{\varepsilon},\left(y_{j}^{\varepsilon}\right)\right) \in P E$. Therefore, $\left(p^{\varepsilon},\left(y_{j}^{\varepsilon}\right)\right) \in A P E$.

Suppose now that $\sum_{j=1}^{n} y_{j}^{\varepsilon} \notin X$ and $\left(p^{\varepsilon},\left(y_{j}^{\varepsilon}\right)\right) \notin \bar{B}(A P E, \varepsilon)$. Since $\left(p^{\varepsilon},\left(y_{j}^{\varepsilon}\right) \in U\right.$ from Claim 1, we then have $x^{\varepsilon} \in z^{\varepsilon}\left(p^{\varepsilon},\left(y_{j}^{\varepsilon}\right)\right)=\left\{\psi\left(p^{\varepsilon},\left(y_{j}^{\varepsilon}\right)\right)\right\} \subset \Psi\left(p^{\varepsilon},\left(y_{j}^{\varepsilon}\right)\right)$. Consequently, $x^{\varepsilon} \in T_{X}\left(\operatorname{proj}_{X}\left(\sum_{j=1}^{n} y_{j}^{\varepsilon}\right)\right)$ and $p^{\varepsilon} \cdot x^{\varepsilon}<0$. From $(2), q \cdot x^{\varepsilon}<0$ for every $q \in S_{\alpha}$, which means that $x^{\varepsilon}$ belongs to the interior of the negative polar cone of $S_{\alpha}$, included in $-\operatorname{int} D$. But $-\operatorname{int} D \cap T_{X}\left(\operatorname{proj}_{X}\left(\sum_{j=1}^{n} y_{j}^{\varepsilon}\right)\right)=\emptyset$ since $\operatorname{proj}_{X}\left(\sum_{j=1}^{n} y_{j}^{\varepsilon}\right) \in \partial X$ and $X+D \subset X$, which leads to a contradiction.

Claim $3 x^{\varepsilon} \in z^{\varepsilon}\left(p^{\varepsilon},\left(y_{j}^{\varepsilon}\right)\right) \cap \mathbb{R e}$. 
Proof: From our choice of $\varepsilon_{0}$ and from Claim 2, $p^{\varepsilon}$ belongs to the relative interior of $S_{\alpha}$. Consequently, (2) implies for $x^{\varepsilon}$ to be in the orthogonal space to the affine space spanned by $S_{\alpha}$, which means that $x^{\varepsilon} \in \mathbb{R} e$. Recalling that $x^{\varepsilon} \in F_{1}^{\varepsilon}\left(x^{\varepsilon}, s^{\varepsilon}, p^{\varepsilon},\left(p_{j}^{\varepsilon}\right)\right)$, we thus have $x^{\varepsilon} \in z^{\varepsilon}\left(p^{\varepsilon},\left(y_{j}^{\varepsilon}\right)\right) \cap \mathbb{R} e$.

\subsubsection{Step 3}

We end the proof of Theorem 2 with a limit argument. We deduce from Step 2 and from the definition of $z^{\varepsilon}$ in Step 1 that, for every $\left.\varepsilon \in\right] 0, \varepsilon_{0}[$, there exists $\left(p^{\varepsilon},\left(y_{j}^{\varepsilon}\right), x^{\varepsilon}, t^{\varepsilon}, u^{\varepsilon}\right) \in S_{\alpha} \times \prod_{j=1}^{n} \partial Y_{j} \times \mathbb{R} e \times[0,1] \times L$ satisfying:

$$
\begin{array}{r}
\left(p^{\varepsilon},\left(y_{j}^{\varepsilon}\right)\right) \in \bar{B}(A P E, \varepsilon), \\
x^{\varepsilon}=t^{\varepsilon} u^{\varepsilon}+\left(1-t^{\varepsilon}\right) \psi\left(p^{\varepsilon},\left(y_{j}^{\varepsilon}\right)\right), \\
t^{\varepsilon}=\tau^{\varepsilon}\left(p^{\varepsilon},\left(y_{j}^{\varepsilon}\right)\right), \\
u^{\varepsilon} \in z\left(\pi\left(p^{\varepsilon}\right),\left(y_{j}^{\varepsilon}\right)\right) .
\end{array}
$$

Furthermore, if $\sum_{j=1}^{n} y_{j}^{\varepsilon} \in X$, then $\left(p^{\varepsilon},\left(y_{j}^{\varepsilon}\right)\right) \in A P E$ and, from the definition of $\tau^{\varepsilon}, t^{\varepsilon}=1$ and $x^{\varepsilon}=u^{\varepsilon} \in z\left(p^{\varepsilon},\left(y_{j}^{\varepsilon}\right)\right)$.

Recall that, for every $\varepsilon \in] 0, \varepsilon_{0}[, \bar{B}(A P E, \varepsilon)$ is in the interior of the compact set $\bar{B}\left(A P E, \varepsilon_{0}\right)$. Consequently, there is a sequence $\left.\left(\varepsilon^{\nu}\right) \subset\right] 0, \varepsilon_{0}$ [ converging to 0 such that the sequence $\left(p^{\varepsilon^{\nu}},\left(y_{j}^{\varepsilon^{\nu}}\right), x^{\varepsilon^{\nu}}, t^{\varepsilon^{\nu}}, u^{\varepsilon^{\nu}}\right)$ converges to an element $\left(p^{*},\left(y_{j}^{*}\right), x^{*}, t^{*}, u^{*}\right)$ in $S_{\alpha} \times \prod_{j=1}^{n} \partial Y_{j} \times \mathbb{R} e \times[0,1] \times L$. We clearly have $\left(p^{*},\left(y_{j}^{*}\right)\right) \in$ $A P E$, hence $\left(p^{*},\left(y_{j}^{*}\right)\right)$ satisfies conditions (a) and (b) of Theorem 2.

We now distinguish two cases. Suppose first that $\sum_{j=1}^{n} y_{j}^{*} \in \operatorname{int} X$. Then, for $\nu$ large enough, $\sum_{j=1}^{n} y_{j}^{\varepsilon^{\nu}} \in \operatorname{int} X$, hence $\left(p^{\varepsilon^{\nu}},\left(y_{j}^{\varepsilon^{\nu}}\right)\right) \in A P E$ and $t^{\varepsilon^{\nu}}=1$. Consequently, $t^{*}=1$ and $x^{*}=u^{*} \in z\left(p^{*},\left(y_{j}^{*}\right)\right)$. We then get $p^{*} \cdot x^{*} \leq 0$ from Assumption (ED), which implies $x^{*} \in-\mathbb{R}_{+} e$ since $x^{*} \in \mathbb{R} e$. We finally have $z\left(p^{*},\left(y_{j}^{*}\right)\right) \cap-\mathbb{R}_{+} e \neq \emptyset:\left(p^{*},\left(y_{j}^{*}\right)\right)$ satisfies condition (c) of Theorem 2.

Suppose now that $\sum_{j=1}^{n} y_{j}^{*} \in \partial X$. From Assumption (ED), $p^{*} \cdot u^{*} \leq 0$, and, from the definition of $\psi, p^{*} \cdot \psi\left(p^{*},\left(y_{j}^{*}\right)\right) \leq 0$, so we clearly have $p^{*} \cdot x^{*} \leq 0$. Consequently, since $x^{*} \in \mathbb{R} e$ and $p^{*} \in S, x^{*} \in-\mathbb{R}_{+} e$. We now end the proof by showing that $x^{*}=u^{*}$. Indeed, if it is not the case, then, from Assumption (ED) and the definition of $\psi, x$ is a convex combination of two elements in $T_{X}\left(\sum_{j=1}^{n} y_{j}^{*}\right)$, one of which belonging to $\operatorname{int} T_{X}\left(\sum_{j=1}^{n} y_{j}^{*}\right)$. Therefore, $x^{*} \in \operatorname{int} T_{X}\left(\sum_{j=1}^{n} y_{j}^{*}\right)$ and $x^{*} \in-\mathbb{R}_{+} e$, which contradicts the fact that $\sum_{j=1}^{n} y_{j}^{*} \in \partial X$. Finally, $x^{*} \in z\left(p^{*},\left(y_{j}^{*}\right)\right) \cap-\mathbb{R}_{+} e:\left(p^{*},\left(y_{j}^{*}\right)\right)$ also satisfies condition (c) of Theorem 2. 


\subsection{Proof of Theorem 1}

Let us first remark that the compacity of the set $A_{0}$ under Assumptions (P), (C) and (BLS) implies the compacity of the subset $K=\left\{\sum_{j=1}^{n} y_{j} \mid\left(y_{j}\right) \in A_{0}\right\}$ of $X$. From Lemma 1 , the set $\left\{\left(\left(x_{i}\right), d\right) \in \prod_{i=1}^{m} X_{i} \times D \mid \sum_{i=1}^{m} x_{i}+d-\omega \in K\right\}$ is compact. Hence, for every $i$, the projection $\widehat{X}_{i}$ of this set on $X_{i}$, i.e. the set of all attainable consumptions for consumer $i$, is compact in $L$. Consequently, from the properties of the preference binary relations $\preceq_{i}$, for every $i$, there exists $x_{i}^{0} \in X_{i}$ such that $x_{i} \prec_{i} x_{i}^{0}$ for every $x_{i} \in \widehat{X}_{i}$.

For every integer $k$, we let $K^{k}=\{x \in L \mid-k e \leq x \leq k e\}$, and, for every $i$, $X_{i}^{k}=X_{i} \cap K^{k}$.

We claim that there exists an integer $k_{0}$ large enough such that, for every $k \geq k_{0}$ and for every $i, x_{i}^{0} \in X_{i}^{k}, \widehat{X}_{i} \subset \operatorname{int} K^{k}$ and

$$
r_{i}\left(p,\left(y_{j}\right)\right)>\inf p \cdot X_{i}^{k} \text { for every }\left(p,\left(y_{j}\right)\right) \in A P E .
$$

Indeed, if it is not true, then there exists a sequence $\left(k^{\nu}, i^{\nu}, p^{\nu},\left(y_{j}^{\nu}\right)\right)$ with values in $\mathbb{N} \times\{1, \ldots, m\} \times A P E$ such that $k^{\nu} \rightarrow \infty$ and $r_{i^{\nu}}\left(p^{\nu},\left(y_{j}^{\nu}\right)\right) \leq \inf p^{\nu} \cdot X_{i^{\nu}}^{k^{\nu}}$ for every $\nu$. We can assume without any loss of generality that the sequence $\left(i^{\nu}\right)$ is constant, say equal to $i$, and that the sequence $\left(p^{\nu},\left(y_{j}^{\nu}\right)\right)$ converges to an element $\left(\bar{p},\left(\bar{y}_{j}\right)\right)$ in $A P E$. Since $r_{i}\left(\bar{p},\left(\bar{y}_{j}\right)\right)>\inf \bar{p} \cdot X_{i}$ from Assumption $(\mathrm{R})$, there exists $x_{i} \in X_{i}$ such that $r_{i}\left(\bar{p},\left(\bar{y}_{j}\right)\right)>\bar{p} \cdot x_{i}$. For $\nu$ large enough, $x_{i} \in X_{i}^{k^{\nu}}$, therefore $p^{\nu} \cdot x_{i} \geq \inf p^{\nu} \cdot X_{i}^{k^{\nu}} \geq r_{i}\left(p^{\nu},\left(y_{j}^{\nu}\right)\right)$. Passing to the limit, one gets $\bar{p} \cdot x_{i} \geq r_{i}\left(\bar{p},\left(\bar{y}_{j}\right)\right)$, which leads to a contradiction.

For every $k \geq k_{0}$ and every $i$, we define the correspondences $\beta_{i}^{k}, \xi_{i}^{k}, \psi_{i}^{k}$ and $f_{i}^{k}$, from $S \times \mathbb{R}$ into $X_{i}^{k}$, as follows:

$$
\begin{aligned}
& \beta_{i}^{k}\left(p, w_{i}\right)=\left\{x_{i} \in X_{i}^{k} \mid p \cdot x_{i} \leq w_{i}\right\}, \\
& \xi_{i}^{k}\left(p, w_{i}\right)=\left\{x_{i} \in \beta_{i}^{k}\left(p, w_{i}\right) \mid x \preceq_{i} x_{i} \text { for every } x \in \beta_{i}^{k}\left(p, w_{i}\right)\right\}, \\
& \psi_{i}^{k}\left(p, w_{i}\right)=\left\{x_{i} \in \xi_{i}^{k}\left(p, w_{i}\right) \mid p \cdot x_{i}=\max p \cdot \xi_{i}^{k}\left(p, w_{i}\right)\right\}, \\
& f_{i}^{k}\left(p, w_{i}\right)= \begin{cases}\psi_{i}^{k}\left(p, w_{i}\right) & \text { if } w_{i}>\inf p \cdot X_{i}^{k}, \\
\left\{x_{i} \in X_{i}^{k} \mid p \cdot x_{i}=\inf p \cdot X_{i}^{k}\right\} & \text { otherwise. }\end{cases}
\end{aligned}
$$

We then define the excess demand correspondence $z^{k}$, from $S \times \prod_{j=1}^{n} \partial Y_{j}$ into $L$, by:

$$
z^{k}\left(p,\left(y_{j}\right)\right)=\sum_{i=1}^{m} f_{i}^{k}\left(p, r_{i}\left(p,\left(y_{j}\right)\right)\right)-\left\{\sum_{j=1}^{n} y_{j}+\omega\right\}
$$

Let us now show that, for every $k \geq k_{0}$, the correspondence $z^{k}$ satisfies Assumption (ED). Indeed, from the properties of the $f_{i}^{k}$ [see Lemma 1 and 2 
in Debreu (1962)], $z^{k}$ is an upper hemi-continuous correspondence with nonempty, convex, compact values. Furthermore, for every $\left(p,\left(y_{j}\right)\right) \in S \times \prod_{j=1}^{n} \partial Y_{j}$, we have:

$$
\begin{aligned}
z^{k}\left(p,\left(y_{j}\right)\right) & =\sum_{i=1}^{m} f_{i}^{k}\left(p, r_{i}\left(p,\left(y_{j}\right)\right)\right)-\left\{\sum_{j=1}^{n} y_{j}+\omega\right\} \\
& \subset \sum_{i=1}^{m} X_{i}^{k}-\left\{\sum_{j=1}^{n} y_{j}+\omega\right\} \\
& \subset X-\left\{\sum_{j=1}^{n} y_{j}\right\} \\
& \subset T_{X}\left(\sum_{j=1}^{n} y_{j}\right) .
\end{aligned}
$$

Finally, we deduce from (4) that, for every $i$,

$$
f_{i}^{k}\left(p, r_{i}\left(p,\left(y_{j}\right)\right)\right)=\psi_{i}^{k}\left(p, r_{i}\left(p,\left(y_{j}\right)\right)\right) \text { for every }\left(p,\left(y_{j}\right)\right) \in A P E .
$$

Hence, for every $\left(p,\left(y_{j}\right)\right) \in A P E$, since $\psi_{i}^{k}\left(p, r_{i}\left(p,\left(y_{j}\right)\right)\right) \subset \beta_{i}^{k}\left(p, r_{i}\left(p,\left(y_{j}\right)\right)\right)$, we have:

$$
\begin{aligned}
\sup p \cdot z^{k}\left(p,\left(y_{j}\right)\right) & =\sum_{i=1}^{m} \sup p \cdot \psi_{i}^{k}\left(p, r_{i}\left(p,\left(y_{j}\right)\right)\right)-p \cdot\left(\sum_{j=1}^{n} y_{j}+\omega\right) \\
& \leq \sum_{i=1}^{m}\left(\sup p \cdot \beta_{i}^{k}\left(p, r_{i}\left(p,\left(y_{j}\right)\right)\right)-r_{i}\left(p,\left(y_{j}\right)\right)\right) \\
& \leq 0
\end{aligned}
$$

Consequently, from Theorem 2 , for every $k \geq k_{0}$, there exists $\left(p^{k},\left(y_{j}^{k}\right)\right) \in$ $S \times \prod_{j=1}^{n} \partial Y_{j}$ such that:

$$
\sum_{j=1}^{n} y_{j}^{k} \in X,\left(p^{k}, \ldots, p^{k}\right) \in \varphi\left(p^{k},\left(y_{j}^{k}\right)\right) \text { and } z^{k}\left(p^{k},\left(y_{j}^{k}\right)\right) \cap-\mathbb{R}_{+} e \neq \emptyset .
$$

Then, from the definition of $z^{k}$, for every $k \geq k_{0}$ there exists $\left(x_{i}^{k}\right) \in \prod_{i=1}^{m} X_{i}^{k}$ and $\rho^{k} \in \mathbb{R}_{+}$such that:

$$
x_{i}^{k} \in f_{i}^{k}\left(p^{k}, r_{i}\left(p^{k},\left(y_{j}^{k}\right)\right)\right) \text { for every } i, \text { and } \sum_{i=1}^{m} x_{i}^{k}=\sum_{j=1}^{n} y_{j}^{k}+\omega-\rho^{k} e
$$

The sequence $\left(\left(x_{i}^{k}\right), p^{k},\left(y_{j}^{k}\right)\right)_{k \geq k_{0}}$ takes its values in the compact set $\prod_{i=1}^{m} \widehat{X}_{i} \times$ $A P E$. Therefore, we can assume without any loss of generality that it converges to an element $\left(\left(x_{i}^{*}\right), p^{*},\left(y_{j}^{*}\right)\right)$ in $\prod_{i=1}^{m} \widehat{X}_{i} \times A P E$.

We end the proof of Theorem 1 by showing that $\left(\left(x_{i}^{*}\right),\left(y_{j}^{*}\right), p^{*}\right)$ is an equilibrium of the economy $\mathcal{E}$.

Since $\left(p^{*},\left(y_{j}^{*}\right)\right) \in A P E$, we have $\left(y_{j}^{*}\right) \in \prod_{j=1}^{n} \partial Y_{j}$ and $\left(p^{*}, \ldots, p^{*}\right) \in \varphi\left(p^{*},\left(y_{j}^{*}\right)\right)$ : condition (b) of Definition 1 is satisfied. 
Since $p^{k} \in \mathcal{H}$ we deduce from (5), (6), (7) and Assumption (R) that:

$$
\begin{aligned}
\rho^{k} & =p^{k} \cdot\left(\rho^{k} e\right) \\
& =p^{k} \cdot\left(\sum_{j=1}^{n} y_{j}^{k}+\omega-\sum_{i=1}^{m} x_{i}^{k}\right) \\
& =p^{k} \cdot\left(\sum_{j=1}^{n} y_{j}^{k}+\omega\right)-\sum_{i=1}^{m} p^{k} \cdot x_{i}^{k} \\
& =\sum_{i=1}^{m}\left(r_{i}\left(p^{k},\left(y_{j}^{k}\right)\right)-p^{k} \cdot x_{i}^{k}\right) \\
& \geq 0 .
\end{aligned}
$$

We now show that $\rho^{k}=0$ for every $k \geq k_{0}$. Suppose it is not true, then $\rho^{k}>0$ and there is a consumer $i$ for which $p^{k} \cdot x_{i}^{k}<r_{i}\left(p^{k},\left(y_{j}^{k}\right)\right)$. Since $x_{i}^{k} \in \widehat{X}_{i}$, we have $x_{i}^{k} \prec_{i} x_{i}^{0}$, and $x_{i}^{k} \in \psi_{i}^{k}\left(p^{k}, r_{i}\left(p^{k},\left(y_{j}^{k}\right)\right)\right.$ then implies $p^{k} \cdot x_{i}^{0}>r_{i}\left(p^{k},\left(y_{j}^{k}\right)\right)$. Hence, from the continuity of the scalar product, there exists $x_{i}^{t}=t x_{i}^{0}+(1-t) x_{i}^{k}$ with $t \in] 0,1\left[\right.$ such that $p^{k} \cdot x_{i}^{t}=r_{i}\left(p^{k},\left(y_{j}^{k}\right)\right)$. From the convexity of $X_{i}^{k}$ and of the binary preference relation $\preceq_{i}$ [Assumption (C)], we deduce that $x_{i}^{t} \in \xi_{i}^{k}\left(p^{k}, r_{i}\left(p^{k},\left(y_{j}^{k}\right)\right)\right)$. Then, since $x_{i}^{k} \in \psi_{i}^{k}\left(p^{k}, r_{i}\left(p^{k},\left(y_{j}^{k}\right)\right)\right.$, we get $p^{k} \cdot x_{i}^{k} \geq$ $p^{k} \cdot x_{i}^{t}=r_{i}\left(p^{k},\left(y_{j} k\right)\right)$, which leads to a contradiction. Finally, passing to the limit in $\sum_{i=1}^{m} x_{i}^{k}=\sum_{j=1}^{n} y_{j}^{k}+\omega-\rho^{k} e$, we get $\sum_{i=1}^{m} x_{i}^{*}=\sum_{j=1}^{n} y_{j}^{*}+\omega$ : condition (c) of Definition 1 is satisfied.

Finally, we prove that condition (a) of Definition 1 is also satisfied. Suppose, on the contrary, that it is not the case. Then there is a consumer $i$ and $x_{i} \in X_{i}$ such that $p^{*} \cdot x_{i} \leq r_{i}\left(p^{*},\left(y_{j}^{*}\right)\right)$ and $x_{i}^{*} \prec_{i} x_{i}$. From the continuity of the binary preference relation $\preceq_{i}$, and since $r_{i}\left(p^{*},\left(y_{j}^{*}\right)\right)>\inf p^{*} \cdot X_{i}$, there exists $x_{i}^{\prime} \in X_{i}$ such that $p^{*} \cdot x_{i}^{\prime}<r_{i}\left(p^{*},\left(y_{j}^{*}\right)\right)$ and $x_{i}^{*} \prec_{i} x_{i}^{\prime}$. Consequently, for $k$ large enough, we have $p^{k} \cdot x_{i}^{\prime}<r_{i}\left(p^{k},\left(y_{j}^{k}\right)\right), x_{i}^{k} \prec_{i} x_{i}^{\prime}$ and $x_{i}^{\prime} \in X_{i}^{k}$, which contradicts the fact that $x_{i}^{k} \in \psi_{i}^{k}\left(p^{k}, r_{i}\left(p^{k},\left(y_{j}^{k}\right)\right)\right) \subset \xi_{i}^{k}\left(p^{k}, r_{i}\left(p^{k},\left(y_{j}^{k}\right)\right)\right)$.

\section{Existence of marginal pricing equilibria}

In this section we shall consider the existence problem of a marginal pricing equilibrium. Firms are said to follow the marginal pricing rule if they fulfill the first order necessary condition for their profit maximization, in the precise mathematical sense formalized by Clarke's normal cone [see Cornet (1989)]. Let us recall the definition of the Clarke's tangent and normal cones [see Clarke $(1975,1983)]$. If $Y$ is a nonempty subset of $L$ and $y$ is an element in $\mathrm{cl} Y$, then Clarke's tangent cone to $Y$ at $y$ is:

$$
T_{Y}(y)=\left\{\begin{array}{l|l}
v \in L & \begin{array}{l}
\forall\left(y^{\nu}\right) \subset Y,\left(y^{\nu}\right) \rightarrow y, \forall\left(t^{\nu}\right) \subset \mathbb{R}_{+}^{*},\left(t^{\nu}\right) \rightarrow 0, \exists\left(v^{\nu}\right) \subset L: \\
\left(v^{\nu}\right) \rightarrow v \text { and } y^{\nu}+t^{\nu} v^{\nu} \in Y \text { for } \nu \text { large enough. }
\end{array}
\end{array}\right\}
$$


Clarke's normal cone to $Y$ at $y$, denoted by $N_{Y}(y)$, is then the negative polar cone of $T_{Y}(y)$.

When $Y$ is closed and convex, Clarke's tangent and normal cones to $Y$ at $y$ reduce to those of convex analysis:

$$
\begin{aligned}
& T_{Y}(y)=\operatorname{cl}\left\{\lambda\left(y^{\prime}-y\right) \mid \lambda \geq 0 \text { and } y^{\prime} \in Y\right\}, \\
& N_{Y}(y)=\left\{p \in L \mid p \cdot y \geq p \cdot y^{\prime} \text { for every } y^{\prime} \in Y\right\} .
\end{aligned}
$$

In the following, we shall denote by $M P$ the marginal pricing rule, the correspondence from $P \times \prod_{j=1}^{n} \partial Y_{j}$ into $\left(e^{+}\right)^{n}$ defined by:

$$
M P\left(p,\left(y_{j}\right)\right)=\prod_{j=1}^{n} N_{Y_{j}}\left(y_{j}\right) .
$$

Remark that, under Assumption (P), the marginal pricing rule $M P$ satifies Assumption (PR) from the properties of the Clarke's normal cone [see Cornet (1989)]. Morover, the free-disposal condition implies that $M P\left(p,\left(y_{j}\right)\right) \backslash\{0\} \subset$ $P^{n}$ for every $\left(p,\left(y_{j}\right)\right) \in P \times \prod_{j=1}^{n} \partial Y_{j}$.

However, the marginal pricing rule does not need to generate bounded losses, unless the production sets are all star-shaped [see Bonnisseau and Cornet (1988)]. We shall thus replace Assumption (BLS) by the following one:

\section{Assumption (BS)}

There exists a real number $t_{0}>\Theta(0)$ such that $A_{t_{0}}$ is nonempty and bounded, and, for every real number $t \in\left[0, t_{0}\right]$ and every $\left(p,\left(y_{j}\right)\right) \in P E$, if $\left(y_{j}\right) \in A_{t}$, then $p \cdot\left(\sum_{j=1}^{n} y_{j}+t e\right)>\inf p \cdot X$.

Even if the marginal pricing rule may generate unbounded losses, we deduce the existence of a marginal pricing equilibrium from Theorem 1. Using an idea of Bonnisseau (1992), we show the existence of a general pricing rule with bounded losses, which coincides with the marginal pricing rule on the set of attainable productions.

Theorem 3 The economy $\mathcal{E}=\left(L,\left(X_{i}, \preceq_{i}, r_{i}\right),\left(Y_{j}\right), M P, \omega\right)$ has an equilibrium under Assumptions (P), $(C),(R)$ and $(B S)$.

Proof: Let us first recall that, under Assumptions (P) and (C), if we let $Y_{0}=-X=-\left(\sum_{i=1}^{m} X_{i}+D-\omega\right)$, then, for every $j=0,1, \ldots, n$, there exists a Lipschitz continuous function $\lambda_{j}: e^{\perp} \rightarrow \mathbb{R}$ satisfying $s_{j}-\lambda_{j}\left(s_{j}\right) e \in \partial Y_{j}$ for every $s_{j} \in e^{\perp}$. Moreover, for every $j=0,1, \ldots, n$, the application $\Lambda_{j}: e^{\perp} \rightarrow$ $\partial Y_{j}$ defined by $\Lambda_{j}\left(s_{j}\right)=s_{j}-\lambda_{j}\left(s_{j}\right) e$ is an homeomorphism [see Lemma 2]. We also recall that the function $\theta$, defined for every $s=\left(s_{j}\right) \in\left(e^{\perp}\right)^{n}$ by $\theta(s)=$ $\sum_{j=1}^{n} \lambda_{j}\left(s_{j}\right)+\lambda_{0}\left(-\sum_{j=1}^{n} s_{j}\right)$, is Lipschitz continuous and that, for every $t \geq 0$, 
the inverse image $M_{t}$ of $A_{t}$ under the application $\Lambda: s \mapsto \Lambda(s)=\left(\Lambda_{j}\left(s_{j}\right)\right)$ satisfies $M_{t}=\left\{s \in\left(e^{\perp}\right)^{n} \mid \theta(s) \leq t\right\}$.

The following generalization of Lemma 4.2 in Bonnisseau and Cornet (1990a) [see the proof in Appendix] shows the link between the generalized gradients of the functions $\lambda_{j}$ and Clarke's normal cones to the production sets.

Lemma 4 Let $Y_{j}$ be a nonempty, closed subset of $L$, different from L, satisfying $Y_{j}-D \subset Y_{j}$, and let $\lambda_{j}$ be the function from $e^{\perp}$ to $\mathbb{R}$ defined in Lemma 2 . Then the generalized gradient of $\lambda_{j}$ at $s \in e^{\perp}$ is given by:

$$
\partial \lambda_{j}\left(s_{j}\right)=\left(N_{Y_{j}}\left(\Lambda_{j}\left(s_{j}\right)\right) \cap S\right)-\left\{\left(1 /\|e\|^{2}\right) e\right\},
$$

and the correspondence $\partial \lambda_{j}$, from $e^{\perp}$ into itself, is upper hemi-continuous with non-empty, convex, compact values.

Consequently, from Clarke (1983) (Propositions 2.3.3 and 2.3.10), for every $s=\left(s_{j}\right) \in\left(e^{\perp}\right)^{n}$ we have:

$$
\partial \theta(s) \subset \Delta(s):=\left\{\begin{array}{l|l}
\left(p_{1}-p, \ldots, p_{n}-p\right) & \begin{array}{l}
p \in-N_{X}\left(-\Lambda_{0}\left(-\sum_{j=1}^{n} s_{j}\right)\right) \cap S \\
\left(p_{j}\right) \in M P(p, \Lambda(s)) \cap S^{n}
\end{array}
\end{array}\right\},
$$

and, from Clarke's normal cone properties, $\Delta$ is an upper hemi-continuous correspondence from $\left(e^{\perp}\right)^{n}$ into itself with non-empty, convex, compact values. Furthermore, from Assumption (BS), $0 \notin \Delta(s)$ for every $s \in\left(e^{\perp}\right)^{n}$ such that $0 \leq \theta(s) \leq t_{0}$.

Since $t_{0}>\Theta(0)$, we have co $M_{0} \subset \operatorname{int} M_{t_{0}}$. Let $r=\inf _{s \notin M_{t_{0}}}\left\|s-\operatorname{proj}_{\mathrm{co} M_{0}}(s)\right\|$ and $\mathcal{M}=\operatorname{co} M_{0}+\frac{r}{2} \bar{B}_{\left(e^{\perp}\right)^{n}}(0,1) . \mathcal{M}$ is a non-empty, convex, compact subset of $\left(e^{\perp}\right)^{n}$ such that $M_{0} \subset \operatorname{int} \mathcal{M}$ and $\mathcal{M} \subset \operatorname{int} M_{t_{0}}$ [see Figure 1].

We can now apply the following topological lemma [see the proof in Appendix], with $a=0$ and $b=t_{0}$.

Lemma 5 Let $E$ be a finite-dimensional Euclidean space, let $a, b \in \mathbb{R}$ such that $a<b$, let $\theta: E \rightarrow \mathbb{R}$ be a locally Lipschitz continuous function, and let $\Delta$ be a correspondence from E into itself. Suppose that:

(i) the set $M_{a b}:=\{s \in E \mid a \leq \theta(s) \leq b\}$ is non-empty and compact;

(ii) $\Delta$ is an upper hemi-continuous correspondence with non-empty, convex, compact values, satisfying $\partial \theta(s) \subset \Delta(s)$ for every $s \in E$;

(iii) $0 \notin \Delta(s)$ for every $s \in M_{a b}$.

If $\mathcal{M}$ is a closed, convex, compact subset of $E$ such that $M_{a} \subset \operatorname{int} \mathcal{M} \subset M_{b}$, then there exists a continuous (homotopy) mapping $H: \partial \mathcal{M} \times[0,1] \rightarrow E$ such that, for every $s \in \partial \mathcal{M}$, 


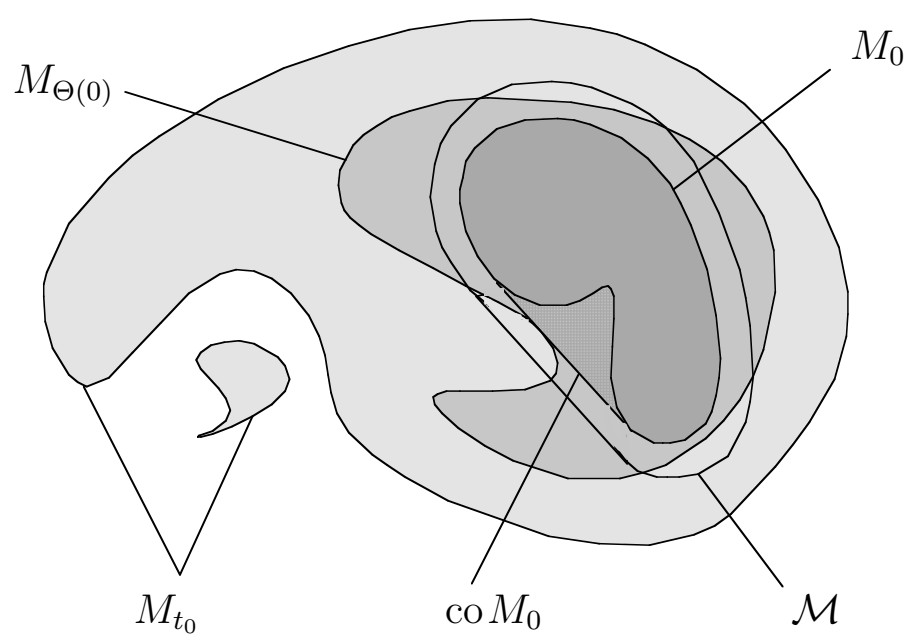

Fig. 1. Geometry of the set $\mathcal{M}$

(I) $\inf H(s, 0) \cdot \Delta(s)>0$;

(II) $H(s, 1) \in-\operatorname{int} T_{\mathcal{M}}(s)$;

(III) $H(s, t) \neq 0$ for every $t \in[0,1]$.

Thus, there exists a continuous mapping $H: \partial \mathcal{M} \times[0,1] \rightarrow\left(e^{\perp}\right)^{n}$ satisfying conditions (I), (II), and (III) of Lemma 5 for every $s \in \partial \mathcal{M}$. Let $\widetilde{H}$ be the mapping from $\partial \mathcal{M} \times[0,1]$ to $\left(e^{\perp}\right)^{n}$ defined by:

$$
\widetilde{H}(s, t)=\frac{H(s, t)}{\max _{j}\left\|H(s, t)_{j}\right\|} .
$$

$\widetilde{H}$ is well defined since $H(s, t) \neq 0$ for every $(s, t) \in \partial \mathcal{M} \times[0,1]$. Moreover, $\widetilde{H}$ satisfies conditions (I), (II), and (III) of Lemma 5: for every $(s, t) \in \partial \mathcal{M} \times[0,1]$,

$$
\begin{array}{r}
\inf \widetilde{H}(s, 0) \cdot \Delta(s)>0 \\
\widetilde{H}(s, 1) \in-\operatorname{int} T_{\mathcal{M}}(s) \\
\widetilde{H}(s, t) \neq 0
\end{array}
$$

We also define the function $\sigma$, from $\left(e^{\perp}\right)^{n}$ to $[0,1]$, by:

$$
\sigma(s)=\left\{\begin{array}{cl}
0 & \text { if } s \in \mathcal{M} \\
\frac{2}{r}\left\|\operatorname{proj}_{\mathcal{M}}(s)-s\right\| & \text { if } s \in \bar{B}_{\left(e^{\perp}\right)^{n}}\left(\mathcal{M}, \frac{r}{2}\right) \backslash \mathcal{M}, \\
1 & \text { if } s \notin \bar{B}_{\left(e^{\perp}\right)^{n}}\left(\mathcal{M}, \frac{r}{2}\right)
\end{array}\right.
$$

where $\operatorname{proj}_{\mathcal{M}}$ denotes the projection mapping onto $\mathcal{M}$. Note that, from the definition of $r$, we have $\bar{B}_{\left(e^{\perp}\right)^{n}}\left(\mathcal{M}, \frac{r}{2}\right)=\bar{B}_{\left(e^{\perp}\right)^{n}}\left(\operatorname{co} M_{0}, r\right) \subset M_{t_{0}}$. 
Finally, for every $\left(p,\left(y_{j}\right)\right) \in P \times \prod_{j=1}^{n} \partial Y_{j}$, let $s=\left(s_{j}\right)=\left(\operatorname{proj}_{e^{\perp}}\left(y_{j}\right)\right)$. Then $\varphi\left(p,\left(y_{j}\right)\right)$ is equal to $M P\left(p,\left(y_{j}\right)\right)$ if $s \in \operatorname{int} \mathcal{M}$, to

$$
\operatorname{co}\left\{M P\left(p,\left(y_{j}\right)\right),\left\{\lambda\left(\frac{1}{p \cdot e}(p, \ldots, p)+\widetilde{H}(s, 0)\right) \mid \lambda \geq 0\right\}\right\}
$$

if $s \in \partial \mathcal{M}$ and to $\left\{\lambda\left(\frac{1}{p \cdot e}(p, \ldots, p)+\widetilde{H}\left(\operatorname{proj}_{\mathcal{M}}(s), \sigma(s)\right)\right) \mid \lambda \geq 0\right\}$ if $s \notin \mathcal{M}$.

We now show that $\varphi$ satisfies Assumption (PR). Since $\widetilde{H}$ and $\sigma$ are continuous and the pricing rule $M P$ satisfies Assumption (PR) under Assumption (P), $\bar{\varphi}$ is an upper hemi-continuous correspondence from $S \times \prod_{j=1}^{n} \partial Y_{j}$ into $\mathcal{H}^{n}$. Moreover, for every $\left(p,\left(y_{j}\right)\right) \in P \times \prod_{j=1}^{n} \partial Y_{j}, \varphi\left(p,\left(y_{j}\right)\right)$ is a nonempty, closed, convex cone of vertex 0 , different from $\{0\}$, and $\varphi\left(\lambda p,\left(y_{j}\right)\right)=\varphi\left(p,\left(y_{j}\right)\right)$ for every $\lambda>0$. Note that $\varphi=M P$ on $M_{0}$ and $M P$ takes their values in $P^{n}$.

Consequently, the correspondence $\varphi$ satisfies Assumption (PR).

From Assumption (BS), there exists $t_{0} \geq 0$ such that $A_{t_{0}}$ is nonempty and bounded. Let us now check that the pricing rule $\varphi$ satisfies parts (BS) and (BL) of Assumption (BLS).

Let $t \in\left[0, t_{0}\left[\right.\right.$ and $\left(p,\left(y_{j}\right)\right) \in S \times \prod_{j=1}^{n} \partial Y_{j}$ such that $(p, \ldots, p) \in \bar{\varphi}\left(p,\left(y_{j}\right)\right)$ and $\sum_{j=1}^{n} y_{j}+t e \in \partial X$. Then $s=\left(\Lambda_{j}^{-1}\left(y_{j}\right)\right) \in \mathcal{M}$ from the construction of $\varphi$ and (10). We now distinguish two cases. Let us first suppose that $s \in$ int $\mathcal{M}$; then $\varphi$ is the marginal pricing rule $M P$, which satisfies Part (BS) of Assumption (BLS) under Assumption (BS).

Suppose now that $s \in \partial \mathcal{M}$. If $p \cdot\left(\sum_{j=1}^{n} y_{j}+t e\right)=\inf p \cdot X$, then $p \in$ $-N_{X}\left(\sum_{j=1}^{n} y_{j}+t e\right)$ and $\sum_{j=1}^{n} y_{j}+t e=-\Lambda_{0}\left(-\sum_{j=1}^{n} s_{j}\right)$. Furthermore, there exists $\left(q_{j}\right) \in \prod_{j=1}^{n}\left(N_{Y_{j}}\left(y_{j}\right) \cap S\right)$ and $\lambda \in[0,1]$ such that $p=\lambda q_{j}+(1-\lambda)(p+$ $\left.\widetilde{H}(s, 0)_{j}\right)$ for every $j$. Hence, $(1-\lambda) \widetilde{H}(s, 0)=-\lambda\left(q_{1}-p, \ldots, q_{n}-p\right)$ with $\left(q_{1}-p, \ldots, q_{n}-p\right) \in \Delta(s)$. Since $0 \notin \Delta(s)$ and $\widetilde{H}(s, 0) \neq 0$, we have $\left.\lambda \in\right] 0,1[$, and $\widetilde{H}(s, 0)=-\frac{\lambda}{1-\lambda} \delta$ with $\delta \in \Delta(s)$. Consequently, $\widetilde{H}(s, 0) \cdot \delta<0$, which contradicts (8). Thus, $\varphi$ satisfies Part (BS) of Assumption (BLS).

Let us now come to part (BL). Let $t \in\left[t_{0}, \Theta\left(t_{0}\right)\right],\left(p,\left(y_{j}\right)\right) \in S \times A_{t}$ such that $p \in-N_{X}\left(\sum_{j=1}^{n} y_{j}+t e\right)$, and let $\left(q_{j}\right) \in \bar{\varphi}\left(p,\left(y_{j}\right)\right)$. From (9), if we let $\hat{s}=s^{\prime}-\varepsilon \widetilde{H}\left(s^{\prime}, 1\right)$ with $s=\left(\Lambda^{-1}\left(y_{j}\right)\right)$ and $s^{\prime}=\operatorname{proj}_{\mathcal{M}}(s)$, then $\hat{s} \in \operatorname{int} \mathcal{M} \subset$ $M_{t_{0}}$ for $\varepsilon>0$ small enough. Let then $\left(\hat{y}_{j}\right)=\Lambda(\hat{s})$; we have $\left(\hat{y}_{j}\right) \in A_{t_{0}}$. Since $\left(y_{j}\right) \in A_{t}$ with $t \geq t_{0}$, we have $s \notin \operatorname{int} \bar{B}_{\left(e^{\perp}\right) n}\left(\mathcal{M}, \frac{r}{2}\right)$, and $\bar{\varphi}\left(p,\left(y_{j}\right)\right)=$ $\left\{(p, \ldots, p)+\widetilde{H}\left(s^{\prime}, 1\right)\right\}$. Consequently, $q_{j}=p+\widetilde{H}\left(s^{\prime}, 1\right)_{j}$ for every $j$, and we can write:

$$
\sum_{j=1}^{n} q_{j} \cdot\left(y_{j}-\hat{y}_{j}\right)=\sum_{j=1}^{n} p \cdot\left(y_{j}-\hat{y}_{j}\right)+\sum_{j=1}^{n} \widetilde{H}\left(s^{\prime}, 1\right)_{j} \cdot\left(y_{j}-\hat{y}_{j}\right) .
$$


We then have:

$$
\sum_{j=1}^{n} q_{j} \cdot y_{j}=\sum_{j=1}^{n} p \cdot y_{j}+\sum_{j=1}^{n}\left(q_{j}-p\right) \cdot \hat{y}_{j}+\sum_{j} \widetilde{H}\left(s^{\prime}, 1\right)_{j} \cdot\left(y_{j}-\hat{y}_{j}\right),
$$

and, since $\widetilde{H}$ takes its values in $\left(e^{\perp}\right)^{n}$ :

$$
\begin{aligned}
\sum_{j=1}^{n} \widetilde{H}\left(s^{\prime}, 1\right)_{j} \cdot\left(y_{j}-\hat{y}_{j}\right) & =\sum_{j=1}^{n} \widetilde{H}\left(s^{\prime}, 1\right)_{j} \cdot\left(s_{j}-\hat{s}_{j}\right) \\
& =\widetilde{H}\left(s^{\prime}, 1\right) \cdot(s-\hat{s}) \\
& =\widetilde{H}\left(s^{\prime}, 1\right) \cdot\left(s-s^{\prime}\right)+\varepsilon\left\|\widetilde{H}\left(s^{\prime}, 1\right)\right\|^{2}
\end{aligned}
$$

From (10), we have $\widetilde{H}\left(s^{\prime}, 1\right) \neq 0$, hence $\varepsilon\left\|\widetilde{H}\left(s^{\prime}, 1\right)\right\|>0$. Moreover, since $s^{\prime}=$ $\operatorname{proj}_{\mathcal{M}}(s), s-s^{\prime} \in N_{\mathcal{M}}(s)$, and (9) implies that $\widetilde{H}\left(s^{\prime}, 1\right) \cdot\left(s-s^{\prime}\right)>0$. Finally, we get $\widetilde{H}\left(s^{\prime}, 1\right) \cdot(s-\hat{s})>0$. Therefore, $\sum_{j=1}^{n} q_{j} \cdot y_{j}>\sum_{j=1}^{n} p \cdot y_{j}+\sum_{j=1}^{n}\left(q_{j}-p\right) \cdot \hat{y}_{j}$ and $\varphi$ satisfies part (BL) of Assumption (BLS).

From Theorem 1 , the economy $\tilde{\mathcal{E}}=\left(L,\left(X_{i}, \preceq_{i}, r_{i}\right),\left(Y_{j}\right), \varphi, \omega\right)$ has an equilibrium and, recalling that, if $\left(p,\left(y_{j}\right)\right) \in S \times A_{0}$, then $s \in M_{0} \subset \operatorname{int} \mathcal{M}$ and $\varphi\left(p,\left(y_{j}\right)\right)=M P\left(p,\left(y_{j}\right)\right)$, every equilibrium of $\tilde{\mathcal{E}}$ is a marginal pricing equilibrium of $\mathcal{E}$.

\section{Existence of quasi-equilibria}

We propose in this section to weaken the survival assumption [part (BS) of Assumption (BLS) in the case of general pricing rules, or Assumption (BS) when the firms follow the marginal pricing rule], by assuming that it is satisfied only for positive real numbers $t \in] 0, t_{0}[$. From the example in Kamiya (1988) we now that there may exists no equilibrium when the survival assumption is not satisfied. However, we show in this section that a quasi-equilibrium can be found when the survival assumption is not satisfied for $t=0$, that is just on the boundary of the attainable allocation, but for $t \in] 0, t_{0}[$.

With respect to the standard Walras equilibrium model, the difference is the same as supposing that the initial endowments are in the interior of the consumption sets or only in the consumption sets. We know that this little difference may have important effects on the equilibrium analysis since a Walras equilibrium may fail to exist under the weak survival assumption, which has the advantage to be more realistic since most of the consumers have an initial endowments in only one commodity, the labor.

Often, the existence of an equilibrium is deduced from a quasi-equilibrium under some irreductibility assumption on the economy. 
Let us first give the definition of a quasi-equilibrium.

Definition 2 A quasi-equilibrium of the economy $\mathcal{E}$ is an element $\left(\left(x_{i}^{*}\right),\left(y_{j}^{*}\right), p^{*}\right)$ in $L^{m} \times L^{n} \times S$ satisfying:

(q-a) for every $i, x_{i}^{*} \in B_{i}\left(p^{*},\left(y_{j}^{*}\right)\right)$ and, for every $x_{i} \in X_{i}$ such that $p^{*} \cdot x_{i}<$ $r_{i}\left(p^{*},\left(y_{j}^{*}\right)\right), x_{i} \preceq_{i} x_{i}^{*} ;$

(b) for every $j, y_{j}^{*} \in \partial Y_{j}$, and $\left(p^{*}, \ldots, p^{*}\right) \in \varphi\left(p^{*},\left(y_{j}^{*}\right)\right)$;

(c) $\sum_{i=1}^{m} x_{i}^{*}=\sum_{j=1}^{n} y_{j}^{*}+\omega$.

Allowing for the survival assumption not to be satisfied for $t=0$ implies that the total wealth in the economy, evaluated at an attainable production equilibrium $\left(p,\left(y_{j}\right)\right) \in A P E$ may be equal to $\inf p \cdot \sum_{i=1}^{m} X_{i}$. We thus have to weaken our Assumption (R) to be consistent with this case.

\section{Assumption ( $\left.\mathbf{R}^{\prime}\right)$}

For every $i, r_{i}$ is continuous on $S \times \prod_{j=1}^{n} \partial Y_{j}$ and satisfies $r_{i}\left(p,\left(y_{j}\right)\right) \geq \inf p \cdot X_{i}$ for every $\left(p,\left(y_{j}\right)\right) \in A P E$. Furthermore, $\sum_{i=1}^{m} r_{i}\left(p,\left(y_{j}\right)\right)=p \cdot\left(\sum_{j=1}^{n} y_{j}+\omega\right)$ for every $\left(p,\left(y_{j}\right)\right) \in P E$.

We also have to slightly strengthen Assumption (PR) to assume that the pricing rule takes its values in $S^{n}$ not only for the attainable allocations but also in a neighborhood. So, we posit:

\section{Assumption (PR)}

For every $\left(p,\left(y_{j}\right)\right) \in P \times \prod_{j=1}^{n} \partial Y_{j}, \varphi\left(p,\left(y_{j}\right)\right)$ is a nonempty, closed, convex cone of vertex 0 , different from $\{0\}$, included in $\left(e^{+}\right)^{n}$, and $\varphi\left(\lambda p,\left(y_{j}\right)\right)=$ $\varphi\left(p,\left(y_{j}\right)\right)$ for every $\lambda>0$. There exists $\bar{t}>0$ such that $\bar{\varphi}\left(p,\left(y_{j}\right)\right) \subset S^{n}$ for every $\left(p,\left(y_{j}\right)\right) \in S \times A_{\bar{t}}$ and $\bar{\varphi}$ is an upper hemi-continuous correspondence on $S \times \prod_{j=1}^{n} \partial Y_{j}$ with nonempty, convex, compact values in $\mathcal{H}^{n}$.

In the general case, Assumption (BLS) will be replaced by the following one:

\section{Assumption (BLS')}

There exists a real number $t_{0}>0$ such that $A_{t_{0}}$ is nonempty and bounded, and:

(BS') for every real number $t \in] 0, t_{0}\left[\right.$ and every $\left(p,\left(y_{j}\right)\right) \in P E$, if $\sum_{j=1}^{n} y_{j}+$ te $\in X$, then $p \cdot\left(\sum_{j=1}^{n} y_{j}+\right.$ te $)>\inf p \cdot X$;

(BL) for every real number $t \in\left[t_{0}, \Theta\left(t_{0}\right)\right]$, every $\left(p,\left(y_{j}\right)\right) \in S \times A_{t}$ and every $\left(q_{j}\right) \in \varphi\left(p,\left(y_{j}\right)\right.$, if $p \in-N_{X}\left(\sum_{j=1}^{n} y_{j}+t e\right)$, then there exists $\left(\hat{y}_{j}\right) \in A_{t_{0}}$ such that $\sum_{j=1}^{n}\left(q_{j}-p\right) \cdot\left(y_{j}-\hat{y}_{j}\right)>0$.

In the particular case of marginal pricing, we shall replace Assumption (BS) by the following: 


\section{Assumption (BS')}

There exists $t_{0}>\Theta(0)$ such that $A_{t_{0}}$ is nonempty and bounded, and, for every real number $\left.t \in] 0, t_{0}\right]$ and every $\left(p,\left(y_{j}\right)\right) \in P E$, if $\sum_{j=1}^{n} y_{j}+t e \in X$, then $p \cdot\left(\sum_{j=1}^{n} y_{j}+t e\right)>\inf p \cdot X$.

Note that the difference comes from the fact that we do not assume that the total wealth of the production sector $\left(p \cdot \sum_{j=1}^{n} y_{j}\right)$ is strictly above the subsistence level of the consumers $(\inf p \cdot X)$ when the total production is on the boundary of $X$.

We can now state the following result.

Theorem 4 The economy $\mathcal{E}=\left(L,\left(X_{i}, \preceq_{i}, r_{i}\right),\left(Y_{j}\right), \varphi, \omega\right)$ has a quasi-equilibrium under Assumptions (P), $(C)$ and ( $R$ ') if $\varphi$ satisfies Assumptions (PR') and $\left(B L S^{\prime}\right)$ or if $\varphi=M P$ satisfies Assumption (BS').

The proof of Theorem 4 is divided into two steps.

\subsection{Step 1}

In this first step, we shall construct auxiliary economies satisfying the conditions of Theorem 1 or of Theorem 3 if $\varphi=M P$.

Let $\nu \in \mathbb{N}^{*}$, large enough so that $1 / \nu<\min \left\{t_{0}, \bar{t}\right\}$, where $t_{0}$ comes from Assumption (BLS') or (BS') and $\bar{t}$ from Assumption (PR'). Let $\omega^{\nu}=\omega+(1 / \nu) e$ and $X^{\nu}:=\sum_{i=1}^{m} X_{i}+D-\omega^{\nu}=X-\{(1 / \nu) e\}$. Under Assumption (C), we can apply Lemma 2 to the set $Y_{0}^{\nu}=-X^{\nu}$ : for every $s \in e^{\perp}$, there exists a unique real number $\lambda_{0}^{\nu}(s)$ such that $s-\lambda_{0}^{\nu}(s) e \in \partial Y_{0}^{\nu}$, and the function $\lambda_{0}^{\nu}$ is Lipschitz continuous on $e^{\perp}$. Note that the definition of $X^{\nu}$ and the unicity property imply that $\lambda_{0}^{\nu}(s)=\lambda_{0}(s)-(1 / \nu)$ for every $s \in e^{\perp}$. For every $s=\left(s_{j}\right) \in\left(e^{\perp}\right)^{n}$, we let $\theta^{\nu}(s):=\sum_{j=1}^{n} \lambda_{j}\left(s_{j}\right)+\lambda_{0}^{\nu}\left(-\sum_{j=1}^{n} s_{j}\right)=\theta(s)-(1 / \nu)$.

For every $t \geq 0$, we let:

$$
A_{t}^{\nu}:=\left\{\left(y_{j}\right) \in \prod_{j=1}^{n} \partial Y_{j} \mid \sum_{j=1}^{n} y_{j} \in X^{\nu}\right\}=A_{t+(1 / \nu)},
$$

and $M_{t}^{\nu}:=\Lambda^{-1}\left(A_{t}^{\nu}\right)=M_{t+(1 / \nu)}$. For every $t \geq 0$, we have:

$$
M_{t}^{\nu}=\left\{s \in\left(e^{\perp}\right)^{n} \mid \theta^{\nu}(s) \leq t\right\},
$$

and we let $\Theta^{\nu}(t):=\max \left\{\theta^{\nu}(s) \mid s \in \operatorname{co} M_{t}^{\nu}\right\}=\Theta(t+(1 / \nu))-(1 / \nu)$. 
We finally let

$$
\begin{gathered}
A P E^{\nu}:=\left\{\left(p,\left(y_{j}\right)\right) \in P E \mid\left(y_{j}\right) \in A_{0}^{\nu}\right\}, \\
\tau^{\nu}=\min \left\{-(1 / \nu), \min _{1 \leq i \leq m}\left\{\inf r_{i}\left(A P E^{\nu}\right)-\inf p \cdot X_{i}\right\}\right\},
\end{gathered}
$$

and, for every $i$ and every $\left(p,\left(y_{j}\right)\right) \in A P E^{\nu}$ :

$$
\begin{aligned}
& \underline{\alpha}_{i}^{\nu}\left(p,\left(y_{j}\right)\right)=\max \left\{\inf p \cdot X_{i}, r_{i}\left(p,\left(y_{j}\right)\right)+m \tau^{\nu}\right\}, \\
& \bar{\alpha}_{i}^{\nu}\left(p,\left(y_{j}\right)\right)=r_{i}\left(p,\left(y_{j}\right)\right)-2 \tau^{\nu} .
\end{aligned}
$$

The following lemma [see the proof in Appendix] gathers some properties of convergence that will be needed in Step 2 when passing to the limit.

Lemma 6 For every $\nu \in \mathbb{N}^{*}, \tau^{\nu}<0$, and the sequence $\left(\tau^{\nu}\right)_{\nu}$ converges to 0 .

We now construct wealth functions $\left(r_{i}^{\nu}\right)$, which satisfies Assumption $(\mathrm{R})$ instead of Assumption ( $\left.\mathrm{R}^{\prime}\right)$. For this, we consider, for every $\nu$ large enough, the correspondences $T_{1}^{\nu}$ and $T_{2}^{\nu}$ from $S \times \prod_{j=1}^{n} \partial Y_{j}$ into $\mathbb{R}^{m}$, defined as follows:

$$
\begin{aligned}
& T_{1}^{\nu}\left(p,\left(y_{j}\right)\right)=\left\{\begin{array}{ll}
\left.\prod_{i=1}^{m}\right] \underline{\alpha}_{i}^{\nu}\left(p,\left(y_{j}\right)\right), \bar{\alpha}_{i}^{\nu}\left(p,\left(y_{j}\right)\right)[ & \text { if }\left(p,\left(y_{j}\right)\right) \in A P E^{\nu} \\
\mathbb{R}^{m} & \text { otherwise }
\end{array},\right. \\
& T_{2}^{\nu}\left(p,\left(y_{j}\right)\right)=\left\{\left(\rho_{i}\right) \in \mathbb{R}^{m} \mid \sum_{i=1}^{m} \rho_{i}=p \cdot\left(\sum_{j=1}^{n} y_{j}+\omega^{\nu}\right)\right\} .
\end{aligned}
$$

The correspondence $T_{1}^{\nu}$ has nonempty, convex values since $\tau^{\nu}<0$ and $r_{i}\left(p,\left(y_{j}\right)\right)-$ $\tau^{\nu} \geq \inf p \cdot X_{i}$ for all $i$ and all $\left(p,\left(y_{j}\right)\right) \in A P E^{\nu}$. Furthermore, $T_{1}^{\nu}$ has an open graph, since $\bar{\alpha}_{i}^{\nu}$ is continuous and $\underline{\alpha}_{i}^{\nu}$ is upper semi-continuous.

$T_{2}^{\nu}$ is lower hemi-continuous with nonempty, convex values. Consequently, their intersection $T^{\nu}=T_{1}^{\nu} \cap T_{2}^{\nu}$ is a lower hemi-continuous correspondence from $S \times \prod_{j=1}^{n} \partial Y_{j}$ into $\mathbb{R}^{m}$ with convex values.

We also show that $T^{\nu}\left(p,\left(y_{j}\right)\right) \neq \emptyset$ for every $\left(p,\left(y_{j}\right)\right) \in S \times \prod_{j=1}^{n} \partial Y_{j}$. It is obvious for every $\left(p,\left(y_{j}\right)\right) \notin A P E^{\nu}$. If $\left(p,\left(y_{j}\right)\right) \in A P E^{\nu}$, then $p \cdot\left(\sum_{j=1}^{n} y_{j}+\right.$ $\left.\omega^{\nu}\right)>\inf p \cdot X$ from Part (BS') of Assumption (BLS') or from Assumption (BS') if $\varphi=M P$, and $\sum_{i=1}^{m} r_{i}\left(p,\left(y_{j}\right)\right)=p \cdot\left(\sum_{j=1}^{n} y_{j}+\omega\right)$ from Assumption $\left(\mathrm{R}^{\prime}\right)$. Since $\tau^{\nu} \leq-\frac{1}{\nu}, p \cdot\left(\sum_{j=1}^{n} y_{j}+\omega^{\nu}\right)<\sum_{i=1}^{m} \bar{\alpha}_{i}^{\nu}\left(p,\left(y_{j}\right)\right)$.

We now show that $\sum_{i=1}^{m} \underline{\alpha}_{i}^{\nu}\left(p,\left(y_{j}\right)\right)<p \cdot\left(\sum_{j=1}^{n} y_{j}+\omega^{\nu}\right)$. If $r_{i}\left(p,\left(y_{j}\right)\right)+m \tau^{\nu} \leq$ $\inf p \cdot X_{i}$ for all $i$, then, recalling that $p \in S$ from Assumption (R'), one has $\inf p \cdot D=0$ and $\inf p \cdot X=\sum_{i=1}^{m} \inf p \cdot X_{i}$. Consequently, $\sum_{i=1}^{m} \underline{\alpha}_{i}^{\nu}\left(p,\left(y_{j}\right)=\right.$ $\sum_{i=1}^{m} \inf p \cdot X_{i}<p \cdot\left(\sum_{j=1}^{n} y_{j}+\omega^{\nu}\right)$. If for some $\hat{1}, r_{\hat{\imath}}\left(p,\left(y_{j}\right)\right)+m \tau^{\nu}>\inf p \cdot X_{\hat{\imath}}$, 
then

$$
\begin{aligned}
\sum_{i=1}^{m} \underline{\alpha}_{i}^{\nu}\left(p,\left(y_{j}\right)\right. & =r_{\hat{\imath}}\left(p,\left(y_{j}\right)\right)+m \tau^{\nu}+\sum_{i \neq \hat{\imath}} \underline{\alpha}_{i}^{\nu}\left(p,\left(y_{j}\right)\right. \\
& =r_{\hat{\imath}}\left(p,\left(y_{j}\right)\right)+\tau^{\nu}+\sum_{i \neq \hat{\imath}}\left(\underline{\alpha}_{i}^{\nu}\left(p,\left(y_{j}\right)+\tau^{\nu}\right) .\right.
\end{aligned}
$$

From the definition of $\tau^{\nu}$, we have $\underline{\alpha}_{i}^{\nu}\left(p,\left(y_{j}\right)+\tau^{\nu} \leq r_{i}\left(p,\left(y_{j}\right)\right)\right.$ for all $i$. Hence $\sum_{i=1}^{m} \underline{\alpha}_{i}^{\nu}\left(p,\left(y_{j}\right) \leq \sum_{i=1}^{m} r_{i}\left(p,\left(y_{j}\right)\right)<p \cdot\left(\sum_{j=1}^{n} y_{j}+\omega^{\nu}\right)\right.$.

From the mean value theorem, there exists a convex combination $\left(\alpha_{i}^{\nu}\left(p,\left(y_{j}\right)\right)\right)$ of $\left(\underline{\alpha}_{i}^{\nu}\left(p,\left(y_{j}\right)\right)\right)$ and $\left(\bar{\alpha}_{i}^{\nu}\left(p,\left(y_{j}\right)\right)\right)$ in $T_{1}^{\nu}\left(p,\left(y_{j}\right)\right)$ such that $\sum_{i=1}^{m} \alpha_{i}^{\nu}\left(p,\left(y_{j}\right)=p\right.$. $\left(\sum_{j=1}^{n} y_{j}+\omega^{\nu}\right)$.

Finally $T^{\nu}$ is a lower hemi-continuous correspondence from $S \times \prod_{j=1}^{n} \partial Y_{j}$ into $\mathbb{R}^{m}$ with nonempty, convex values. From Michael's theorem, there exists a continuous selection $r^{\nu}=\left(r_{i}^{\nu}\right)$ of $T^{\nu}$, satisfying:

$$
\sum_{i=1}^{m} r_{i}^{\nu}\left(p,\left(y_{j}\right)\right)=p \cdot\left(\sum_{j=1}^{n} y_{j}+\omega^{\nu}\right) \text { for every }\left(p,\left(y_{j}\right)\right) \in P E
$$

and, for every $i$ :

$$
r_{i}^{\nu}\left(p,\left(y_{j}\right)\right)>\inf p \cdot X_{i} \text { for every }\left(p,\left(y_{j}\right)\right) \in A P E^{\nu},
$$

Let us now consider the auxiliary economy $\mathcal{E}^{\nu}=\left(\left(X_{i}, \preceq_{i}, r_{i}^{\nu}\right),\left(Y_{j}\right), \varphi, \omega^{\nu}\right)$. Assumptions (P) and (C) are clearly satisfied in $\mathcal{E}^{\nu}$. Assumption (R) is also satisfied in $\mathcal{E}^{\nu}$ from (11), (12) and the continuity of $r^{\nu}$.

Let us first consider the case of a general pricing rule. When $1 / \nu \leq \bar{t}, \mathcal{E}^{\nu}$ satisfies Assumption (PR) since $\mathcal{E}$ satisfies Assumption (PR). Let us now come to Assumpton (BLS). If we let $t_{0}^{\nu}=t_{0}-(1 / \nu)$, then $t_{0}^{\nu} \geq 0$ since $1 / \nu \leq \bar{t}_{0}$, and $A_{t_{0}^{\nu}}^{\nu}=A_{t_{0}}$ is nonempty and bounded. For every $t \in\left[0, t_{0}^{\nu}\right.$ [ and every $\left(p,\left(y_{j}\right)\right) \in P E$, if $\sum_{j=1}^{n} y_{j}+t e \in X^{\nu}$, then $\left(y_{j}\right) \in A_{t}^{\nu}=A_{t+(1 / \nu)}$ with $t+(1 / \nu) \in$ $\left[(1 / \nu), t_{0}^{\nu}[\subset] 0, t_{0}\left[\right.\right.$. We then deduce from Assumption (BLS') that $p \cdot\left(\sum_{j=1}^{n} y_{j}+\right.$ $(t+(1 / \nu)) e))>\inf p \cdot X$, which clearly implies $p \cdot\left(\sum_{j=1}^{n} y_{j}+t e\right)>\inf p \cdot X^{\nu}=$ $\inf p \cdot X-(1 / \nu)$. For every $t \in\left[t_{0}^{\nu}, \Theta^{\nu}\left(t_{0}^{\nu}\right)\right]$, every $\left(p,\left(y_{j}\right)\right) \in S \times A_{t}^{\nu}$ and every $\left(q_{j}\right) \in \bar{\varphi}\left(p,\left(y_{j}\right)\right)$, if $p \in-N_{X^{\nu}}\left(\sum_{j=1}^{n} y_{j}+t e\right)$, then $p \in-N_{X}\left(\sum_{j=1}^{n} y_{j}+(t+\right.$ $(1 / \nu)) e)$ with $\left(y_{j}\right) \in A_{t+(1 / \nu)}$ and $t+(1 / \nu) \in\left[t_{0}^{\nu}+(1 / \nu), \Theta^{\nu}\left(t_{0}^{\nu}\right)+(1 / \nu)\right]=$ $\left[t_{0}, \Theta\left(t_{0}\right)\right]$. We then deduce from Assumption (BLS') that there exists $\left(\hat{y}_{j}\right) \in$ $A_{t_{0}}=A_{t_{0}^{\nu}}^{\nu}$ such that $\sum_{j=1}^{n}\left(q_{j}-p\right) \cdot\left(y_{j}-\hat{y}_{j}\right)>0$.

In the case where $\varphi=M P$ and Assumption (BS') is satisfied in the economy $\mathcal{E}$, Assumption (BS) is also satisfied in the economy $\mathcal{E}^{\nu}$ for $\nu$ such that $t_{0}^{\nu}=$ $t_{0}-(1 / \nu)>\Theta^{\nu}(0)=\Theta(1 / \nu)-(1 / \nu)$. We first show that $t_{0}^{\nu}>\Theta^{\nu}(0)$ for $\nu$ large enough. Suppose on the contrary that $t_{0}^{\nu} \leq \Theta^{\nu}(0)$, i.e. that $t_{0} \leq$ $\Theta(1 / \nu)$ for every $\nu \in \mathbb{N}^{*}$. For $\nu$ large enough, $M_{1 / \nu}$ is a subset of $M_{t_{0}}$, which is compact under Assumptions (P), (C) and (BS'). From the definition of 
the function $\Theta$, there exists $s^{\nu} \in \operatorname{co} M_{1 / \nu}$ such that $\Theta(1 / \nu)=\theta\left(s^{\nu}\right)$. From Carathodory's theorem, there exists then $m(\ell-1)+1$ vectors $\left(s_{k}^{\nu}\right)$ in $M_{1 / \nu}$ and $\lambda^{\nu}$ in the simplex of $\mathbb{R}^{m(\ell-1)+1}$, such that $s^{\nu}=\sum_{k} \lambda_{k}^{\nu} s_{k}^{\nu}$. From the compacity of the $M_{t_{0}}$ and the compacity of the simplex, we can suppose without any loss of generality that, the sequence $\left(s_{k}^{\nu}\right)_{\nu}$ converges to $\left(\bar{s}_{k}\right) \in M_{0}$ and that the sequence $\left(\lambda^{\nu}\right)_{\nu}$ converges to $\bar{\lambda}$. Consequently, the sequence $\left(s^{\nu}\right)_{\nu}$ converges to $\bar{s}:=\sum_{k} \bar{\lambda}_{k} \bar{s}_{k} \in \operatorname{co} M_{0}$. Since the function $\theta$ is continuous, passing to the limit in $t_{0} \leq \theta\left(s^{\nu}\right)$ we get $t_{0} \leq \theta(\bar{s})$, which contradicts the fact that $\Theta(0)<t_{0}$. Consequently, for $\nu$ large enough, we have $t_{0}^{\nu}>\Theta^{\nu}(0)$.

Note that $A_{t_{0}^{\nu}}^{\nu}=A_{t_{0}}$ is nonempty and bounded. Furthermore, for every $t \in$ $\left[0, t_{0}^{\nu}\right]$ and every $\left(p,\left(y_{j}\right)\right) \in P E$, if $\left(y_{j}\right) \in A_{t}^{\nu}$, then $\left(y_{j}\right) \in A_{t+(1 / \nu)}$ with $t+$ $\left.(1 / \nu) \in] 0, t_{0}\right]$, and we deduce from Assumption (BS') that $p \cdot \sum_{j=1}^{n} y_{j}+(t+$ $(1 / \nu)) e)>\inf p \cdot X$, which implies $p \cdot\left(\sum_{j=1}^{n} y_{j}+t\right)>\inf p \cdot X^{\nu}$.

Hence, for $\nu$ large enough, from Theorems 1 and 3, there exists an equilibrium in the economy $\mathcal{E}^{\nu}$, i.e. there exists $\left(\left(x_{i}^{\nu}\right),\left(y_{j}^{\nu}\right), p^{\nu}\right)$ in $L^{m} \times L^{n} \times S$ such that, if we let $B_{i}^{\nu}\left(p^{\nu},\left(y_{j}^{\nu}\right)\right)=\left\{x_{i} \in X_{i} \mid p^{\nu} \cdot x_{i} \leq r_{i}^{\nu}\left(p^{\nu},\left(y_{j}^{\nu}\right)\right)\right\}$, then:

$$
\begin{array}{r}
\text { for every } i, x_{i}^{\nu} \text { is a greater element for } \preceq_{i} \text { in } B_{i}^{\nu}\left(p^{\nu},\left(y_{j}^{\nu}\right)\right), \\
\text { for every } j, y_{j}^{\nu} \in \partial Y_{j} \text { and }\left(p^{\nu}, \ldots, p^{\nu}\right) \in \bar{\varphi}\left(p^{\nu},\left(y_{j}^{\nu}\right)\right), \\
\sum_{i=1}^{m} x_{i}^{\nu}=\sum_{j=1}^{n} y_{j}^{\nu}+\omega+\frac{1}{\nu} e .
\end{array}
$$

\subsection{Step 2}

In this section we show that the sequence of equilibria of the economies $\mathcal{E}^{\nu}$ converges to a quasi-equilibrium of the initial economy $\mathcal{E}$.

From (15) we deduce that, for $\nu$ large enough, $\left(\left(x_{i}^{\nu}\right),\left(y_{j}^{\nu}\right), p^{\nu}\right)$ belongs to the set $\widehat{X}_{t_{0}} \times A_{t_{0}} \times S$, where $\widehat{X}_{t_{0}}=\left\{\left(x_{i}\right) \in \prod_{i=1}^{m} X_{i} \mid \exists d \in D: \sum_{i=1}^{m} x_{i}+d-\omega \in K_{t_{0}}\right\}$, with $K_{t_{0}}=\left\{\sum_{j=1}^{n} y_{j}+t_{0} e \mid\left(y_{j}\right) \in A_{t_{0}}\right\} \subset X$. Under Assumptions (P), (C) and (BLS') or (BS'), if $\varphi=M P$ the set $A_{t_{0}}$ is compact, and $K_{t_{0}}$ is a compact subset of $X$. From Lemma $1, \widehat{X}_{t_{0}}$ is compact, and we can suppose without any loss of generality that the sequence $\left(\left(x_{i}^{\nu}\right),\left(y_{j}^{\nu}\right), p^{\nu}\right)_{\nu}$ converges to an element $\left(\left(x_{i}^{*}\right),\left(y_{j}^{*}\right), p^{*}\right) \in \widehat{X}_{t_{0}} \times A_{t_{0}} \times S$. Let us now check that $\left(\left(x_{i}^{*}\right),\left(y_{j}^{*}\right), p^{*}\right)$ is a quasi-equilibrium of $\mathcal{E}$.

Passing to the limit in (15), we get: $\sum_{i=1}^{m} x_{i}^{*}=\sum_{j=1}^{n} y_{j}^{*}+\omega:\left(\left(x_{i}^{*}\right),\left(y_{j}^{*}\right), p^{*}\right)$ satisfies condition (c) of Definition 1.

For every $j$, since $Y_{j}$ is closed, $y_{j}^{*} \in \partial Y_{j}$. Furthermore, since $\bar{\varphi}$ is an up- 
per hemi-continuous correspondence [under Assumption (P) if $\varphi=M P$; under Assumption (PR) otherwise], from (14) we get $\left(p^{*}, \ldots, p^{*}\right) \in \bar{\varphi}\left(p^{*},\left(y_{j}^{*}\right)\right)$ : $\left(\left(x_{i}^{*}\right),\left(y_{j}^{*}\right), p^{*}\right)$ satisfies condition (b) of Definition 2.

For every $i$, since $X_{i}$ is closed, $x_{i}^{*} \in X_{i}$. Furthermore, for every $i$, since $x_{i}^{\nu} \in B_{i}^{\nu}\left(p^{\nu},\left(y_{j}^{\nu}\right)\right)$ for $\nu$ large enough, $\left(p^{\nu},\left(y_{j}^{\nu}\right)\right) \in A P E^{\nu}$ and $r_{i}^{\nu}\left(p,\left(y_{j}\right)\right)<$ $\bar{\alpha}_{i}^{\nu}\left(p,\left(y_{j}\right)\right)$ for every $\left(p,\left(y_{j}\right)\right) \in A P E^{\nu}$ by construction, we deduce that $p^{\nu}$. $x_{i}^{\nu}<r_{i}\left(p^{\nu},\left(y_{j}^{\nu}\right)\right)-2 \tau^{\nu}$ for $\nu$ large enough. From the continuity of $r_{i}$ [under Assumption $\left.\left(\mathrm{R}^{\prime}\right)\right]$ and the fact that the sequence $\left(\tau^{\nu}\right)_{\nu}$ converges to 0 (Lemma 6), we finally get $p^{*} \cdot x_{i}^{*} \leq r_{i}\left(p^{*},\left(y_{j}^{*}\right)\right)$, that is $x_{i} \in B_{i}\left(p^{*},\left(y_{j}^{*}\right)\right)$. Suppose now that there is a consumer $i$ for which there exists $x_{i} \in X_{i}$ such that $p^{*} \cdot x_{i}<r_{i}\left(p^{*},\left(y_{j}^{*}\right)\right)$ and $x_{i}^{*} \prec_{i} x_{i}$. Then, from the continuity of $r_{i}$ and $\preceq_{i}$ and from the fact that the sequence $\left(\tau^{\nu}\right)_{\nu}$ converges to 0 , for $\nu$ large enough we have $p^{\nu} \cdot x_{i}<r_{i}\left(p^{\nu},\left(y_{j}^{\nu}\right)\right)+m \tau^{\nu}<r_{i}^{\nu}\left(p^{\nu},\left(y_{j}^{\nu}\right)\right)$ and $x_{i}^{\nu} \prec_{i} x_{i}$, which contradicts (13). Thus, $\left(\left(x_{i}^{*}\right),\left(y_{j}^{*}\right), p^{*}\right)$ satisfies condition (q-a) of Definition 2.

\section{Appendix: Lemmata}

Proof of Lemma 1: First of all, remark that, since $D$ and the consumption sets $X_{i}$ are nonempty and convex from Assumption (C), the set $X=\sum_{i=1}^{m} X_{i}+D-\omega$ is nonempty and convex. Moreover, it clearly satisfies $X+D=X$.

Let us now show that $X$ is closed. From Assumption (C), the cones $\mathcal{A} X_{i}$, for all $i$, and $D$ are included in $e^{+}$. So, one easily checks that they are positively semiindependant, and this implies that $X=\sum_{i=1}^{m} X_{i}+D-\omega$ is closed [see (9) in Paragraph 1.9 in Debreu (1959)].

Suppose now that there is a consumer $i$ for which inf $e \cdot X_{i}=-\infty$. Then there exists a sequence $\left(x_{i}^{\nu}\right)_{\nu}$ taking its values in $X_{i}$ satisfying $e \cdot x_{i} \leq 0$ for every $\nu$ and such that the sequence $\left(\left\|x_{i}^{\nu}\right\|\right)_{\nu}$ converges to $+\infty$. For every $\nu$, let $\xi_{i}^{\nu}=\frac{x_{i}^{\nu}}{\left\|x_{i}^{\nu}\right\|}$. The sequence $\left(\xi_{i}^{\nu}\right)$ clearly takes its values in the compact subset $\mathcal{A} X_{i} \cap \bar{B}_{L}(0,1)$. We can thus suppose without any loss of generality that it converges to some $\xi_{i} \in \mathcal{A} X_{i} \cap \bar{B}_{L}(0,1)$. From $\left\|\xi_{i}^{\nu}\right\|=1$ we deduce that $\left\|\xi_{i}\right\|=1$, hence $\xi_{i} \neq 0$. From $\xi_{i} \in \mathcal{A} X_{i}$, since $\mathcal{A} X_{i} \subset e^{+}$, we finally deduce that $e \cdot \xi_{i}>0$. But, since $e \cdot x_{i}^{\nu} \leq 0$ for every $\nu$, we get $e \cdot \xi_{i} \leq 0$, which is a contradiction. Consequently, we have $t_{i}=\inf e \cdot X_{i}>-\infty$ for every $i$, i.e. $X_{i} \subset\left\{t_{i} e\right\}+e^{+}$for every $i$. Hence, $X=\sum_{i=1}^{m} X_{i}+D-\omega \subset\left\{\sum_{i=1}^{m} t_{i}-\omega\right\}+e^{+}$, and $X \neq L$.

Let now $K$ be a compact subset of $X$. In order to show that the set $A_{K}=\left\{\left(\left(x_{i}\right), d\right) \in\right.$ $\left.\prod_{i=1}^{m} X_{i} \times D \mid \sum_{i=1}^{m} x_{i}+d-\omega \in K\right\}$ is compact, we shall first prove that it is bounded. Let $\left(\left(x_{i}^{\nu}\right), d^{\nu}\right)$ be a sequence in $\prod_{i=1}^{m} X_{i} \times D$ such that $\sum_{i=1}^{m} x_{i}^{\nu}+d^{\nu}-\omega=$ $k^{\nu} \in K$ for every $\nu$. Suppose that $\left\|\left(\left(x_{i}^{\nu}\right), d^{\nu}\right)\right\|_{\infty}=\max _{2}\left\{\max _{i}\left\{\left\|x_{i}^{\nu}\right\|\right\},\left\|d^{\nu}\right\|\right\}$ tends

to $+\infty$. For every $\nu$, let $\xi_{i}^{\nu}=\frac{x_{i}^{\nu}}{\left\|\left(\left(x_{i}^{\nu}\right), d^{\nu}\right)\right\|_{\infty}}$ for every $i$ and $\delta^{\nu}=\frac{d^{\nu}}{\left\|\left(\left(x_{i}^{\nu}\right), d^{\nu}\right)\right\|_{\infty}}$. Since $\left\|\left(\left(\xi_{i}^{\nu}\right), \delta^{\nu}\right)\right\|_{\infty}=1$ for every $\nu$, we can suppose that the sequence $\left(\left(\xi_{i}\right), \delta^{\nu}\right)$ converges 
to some $\left(\left(\xi_{i}\right), \delta\right) \in L^{m} \times D$ satisfying $\left\|\left(\left(\xi_{i}\right), \delta\right)\right\|=1$. Note that $\xi_{i} \neq 0$ implies that $\left\|x_{i}^{\nu}\right\|$ tends to $+\infty$, and consequently, $\xi_{i} \in \mathcal{A} X_{i}$.

Since $K$ is compact and $k^{\nu} \in K$ for every $\nu$, passing to the limit in $\sum_{i=1}^{m} \xi_{i}^{\nu}+\delta^{\nu}-$ $\frac{\omega}{\left\|\left(\left(x_{i}^{\nu}\right), d^{\nu}\right)\right\|_{\infty}}=\frac{k^{\nu}}{\left\|\left(\left(x_{i}^{\nu}\right), d^{\nu}\right)\right\|_{\infty}}$ we get $\sum_{i=1}^{m} \xi_{i}+\delta=0$, which implies that $\xi_{i}=0$ for every $i$ and $\delta=0$ from the positive semi-independence of the cones $\mathcal{A} X_{1}, \ldots, \mathcal{A} X_{M}, D$. Finally, $\left(\left(\xi_{i}\right), \delta\right)=0$ contradicts the fact that $\left\|\left(\left(\xi_{i}\right), \delta\right)\right\|=1$.

We now finish the proof by showing that $A_{K}$ is closed. Let $\left(\left(x_{i}^{\nu}\right), d^{\nu}\right)$ be a sequence in $\prod_{i=1}^{m} X_{i} \times D$ converging to $\left(\left(x_{i}\right), d\right)$, and, such that $\sum_{i=1}^{m} x_{i}^{\nu}+d^{\nu}-\omega=k^{\nu} \in K$ for every $\nu$. Since the sets $X_{i}$ and $D$ are closed, $\left(\left(x_{i}\right), d\right) \in \prod_{i=1}^{m} X_{i} \times D$. Furthermore, since $K$ is compact, $\left(k^{\nu}\right)$ has a sub-sequence, which converges to $k \in K$. Thus, $\sum_{i=1}^{m} x_{i}+d-\omega=k$ and $\left(\left(x_{i}\right), d\right) \in A_{K}$.

Proof of Lemma 2: We shall omit the subscript $j$ for the sake of clarity.

We first establish that, for every $s \in e^{\perp}$, there exists a real number $\lambda$ such that $s-\lambda e \in \partial Y$. Indeed, let $y^{\prime}, y^{\prime \prime}$ be elements in $L$ such that $y^{\prime} \in Y$ and $y^{\prime \prime} \notin Y$. Let $t \geq-\min \left\{p \cdot\left(y^{\prime}-s\right) \mid p \in S\right\}$. Then $s-t e \in Y$. Indeed, it suffices to show from the free-disposal assumption that $s-t e \in y^{\prime}-D$ or equivalently that $y^{\prime}-s+t e \in D$. From the Bipolar Theorem [see Theorem 14.5 in Rockafellar (1970)], this is true if for all $p \in S, p \cdot\left(y^{\prime}-s+t e\right) \geq 0$. This last condition holds true from our choice of $t$.

Let $t<\min \left\{p \cdot\left(s-y^{\prime \prime}\right) \mid p \in S\right\}$. Then $s-t e \notin Y$. Indeed, it suffices to show from the free-disposal assumption that $s-t e \in y^{\prime \prime}+\operatorname{int} D$ or equivalently that $s-y^{\prime \prime}-t e \in \operatorname{int} D$. This is true if for all $p \in S, p \cdot\left(s-y^{\prime \prime}-t e\right)>0$. This last condition holds true from our choice of $t$.

Finally, the set $\{t \in \mathbb{R} \mid s-t e \in Y\}$ is non-empty and bounded below. We let $\lambda=\inf \{t \in \mathbb{R} \mid s-t e \in Y\}$. One checks that $s-\lambda e \in \partial Y$ since $e \in \operatorname{int} D$.

We now show simultaneously the uniqueness part and the fact that the function $\lambda$ is Lipschitz continuous. Let $s_{1}, s_{2}$ be elements in $e^{\perp}$ and let $\lambda_{1}$ and $\lambda_{2}$ such that $y_{1}=s_{1}-\lambda_{1} e \in \partial Y$ and $y_{2}=s_{2}-\lambda_{2} e \in \partial Y$. Without any loss of generality, we can assume that $\lambda_{1} \geq \lambda_{2}$. Suppose that $y_{1}-y_{2} \in-\operatorname{int} D$. Then $y_{1} \in y_{2}-\operatorname{int} D \subset \operatorname{int} Y$, which contradicts the fact that $y_{1} \in \partial Y$. Thus, we have $y_{1}-y_{2} \notin-\operatorname{int} D$, and there exists $p \in S$ such that $p \cdot\left(y_{1}-y_{2}\right) \geq 0$. This implies $p \cdot\left(s_{1}-s_{2}\right)+\lambda_{2}-\lambda_{1} \geq 0$ since $p \cdot e=1$. Hence:

$$
\left|\lambda_{1}-\lambda_{2}\right|=\lambda_{1}-\lambda_{2} \leq p \cdot\left(s_{1}-s_{2}\right) \leq \max \left\{q \cdot\left(s_{1}-s_{2}\right) \mid q \in S\right\} .
$$

The function from $e^{\perp}$ to $\mathbb{R}$, which associates $\max \{q \cdot \sigma \mid q \in S\}$ to $\sigma$, is a finite, convex, homogeneous of degree 1 function. Consequently, it is locally Lipschitz continuous at 0 and with the homogeneity, one deduces that it is Lipschitz continuous on $e^{\perp}$. Consequently, there exists $k \geq 0$ such that $\max \{q \cdot \sigma \mid q \in S\} \leq k\|\sigma\|$ for all $\sigma \in e^{\perp}$. Finally, one gets $\left|\lambda_{1}-\lambda_{2}\right| \leq k\left\|s_{1}-s_{2}\right\|$.

This shows firstly that, for every $s \in e^{\perp}$, the element $\lambda(s)$ is uniquely defined since $s_{1}=s_{2}$ implies that $\lambda_{1}=\lambda_{2}$. Secondly, this shows that the function $\lambda: e^{\perp} \rightarrow \mathbb{R}$ is 
Lipschitz continuous of rank $k$.

Let us now establish the fact that $Y=\left\{y \in L \mid \lambda\left(\operatorname{proj}_{e^{\perp}}(y)\right)+\left(y \cdot e /\|e\|^{2}\right) \leq 0\right\}$. Indeed, if $y \in Y$, then one has $y=\operatorname{proj}_{e^{\perp}}(y)+\left(y \cdot e /\|e\|^{2}\right) e$, and the definition of $\lambda$ implies that $\lambda\left(\operatorname{proj}_{e^{\perp}}(y)\right) \leq-\left(y \cdot e /\|e\|^{2}\right)$. Conversely, if $\lambda\left(\operatorname{proj}_{e^{\perp}}(y)\right)+\left(y \cdot e /\|e\|^{2}\right) \leq$ 0 , then one has

$$
y=\operatorname{proj}_{e^{\perp}}(y)+\frac{y \cdot e}{\|e\|^{2}} e=\operatorname{proj}_{e^{\perp}}(y)-\lambda\left(\operatorname{proj}_{e^{\perp}}(y)\right) e+\left(\lambda\left(\operatorname{proj}_{e^{\perp}}(y)\right)+\frac{y \cdot e}{\|e\|^{2}}\right) e .
$$

Since $\operatorname{proj}_{e^{\perp}}(y)-\lambda\left(\operatorname{proj}_{e^{\perp}}(y)\right) e \in \partial Y$ and $Y-\mathbb{R}_{+} e \subset Y$, this implies that $y \in Y$.

We end the proof by showing that the mapping $\Lambda: s \rightarrow s-\lambda(s) e$ has $\operatorname{proj}_{e^{\perp} \mid \partial Y}$, the restriction to $\partial Y$ of the orthogonal projection onto $e^{\perp}$, for inverse. One clearly has $\operatorname{proj}_{e^{\perp}}(s-\lambda(s) e)=s$ for every $s \in e^{\perp}$, and we now show that $\operatorname{proj}_{e^{\perp}}(y)-$ $\lambda\left(\operatorname{proj}_{e^{\perp}}(y)\right) e=y$ for every $y \in \partial Y$. Indeed, one has $y=\operatorname{proj}_{e^{\perp}}(y)-t e$ with $t=-\frac{y \cdot e}{\|e\|^{2}}$ and the above uniqueness property and the fact that $y \in \partial Y$ imply that $\lambda\left(\operatorname{proj}_{e^{\perp}}(y)\right)=t$.

Proof of Lemma 3: Let us first show part (BL) of Lemma 3. If for all $\gamma>0$, part (BL) is false, then there exists a sequence $\left(p^{\nu}, s^{\nu}\right)_{\nu}$ in $S \times\left(e^{\perp}\right)^{n}$ and a sequence $\left(q_{j}^{\nu}\right)_{\nu}$ in $S^{n}$ such that, for every $\nu, s^{\nu} \in \mathcal{B}_{\underline{\underline{1}}} \backslash \operatorname{int} M_{t_{0}}, p^{\nu} \in-N_{X}\left(\operatorname{proj}_{X}\left(\sum_{j=1}^{n} \Lambda_{j}\left(s_{j}^{\nu}\right)\right)\right)$, $\left(q_{j}^{\nu}\right) \in \bar{\varphi}\left(p^{\nu}, \Lambda\left(s^{\nu}\right)\right)$ and $\sum_{j=1}^{n}\left(q_{j}^{\nu}-p^{\nu}\right)^{\nu} \cdot\left(\Lambda_{j}\left(s_{j}^{\nu}\right)-\hat{y}_{j}\right) \leq 0$ for every $\left(\hat{y}_{j}\right) \in A_{t_{0}}$. Since the sequence $\left(p^{\nu}, s^{\nu}\right)_{\nu}$ is bounded, we can suppose without any loss of generality that it converges to some element $(p, s) \in S \times\left(e^{\perp}\right)^{n}$. From $s^{\nu} \in \mathcal{B}_{\underline{1}} \backslash \operatorname{int} M_{t_{0}}$, we deduce that $s \in \mathcal{B}_{0}=\operatorname{co} M_{t_{0}}$ and $s \notin \operatorname{int} M_{t_{0}}$. From $p^{\nu} \in-N_{X}\left(\operatorname{proj}_{X}\left(\sum_{j=1}^{n} \Lambda_{j}\left(s_{j}^{\nu}\right)\right)\right)$, we deduce that $p \in-N_{X}\left(\operatorname{proj}_{X}\left(\sum_{j=1}^{n} \Lambda_{j}\left(s_{j}\right)\right)\right)$, hence $p \in-N_{X}\left(\sum_{j=1}^{n} \Lambda_{j}\left(s_{j}\right)+t e\right)$ with $t \in\left[t_{0}, \Theta\left(t_{0}\right)\right]$ since $s \in \operatorname{co} M_{t_{0}} \backslash \operatorname{int} M_{t_{0}}$. From $\left(q_{j}^{\nu}\right) \in \bar{\varphi}\left(p^{\nu}, \Lambda\left(s^{\nu}\right)\right)$ and the upper hemi-continuity of the normalized general pricing rule $\bar{\varphi}$, we can suppose that the sequence $\left(q_{j}^{\nu}\right)$ converges to some $\left(q_{j}\right) \in \bar{\varphi}(p, \Lambda(s))$. We finally deduce that $\sum_{j=1}^{n}\left(q_{j}-\right.$ $p) \cdot\left(\Lambda_{j}\left(s_{j}\right)-\hat{y}_{j}\right) \leq 0$ for every $\left(\hat{y}_{j}\right) \in A_{t_{0}}$, which contradicts Assumption (BLS).

Part (BS) of Lemma 3 is a consequence of Part (BS) of Assumption (BLS) and of part (BL) of the lemma [see Remark 3].

Proof of Lemma 4: We first remark that, from Lemma 2, we have:

$$
Y_{j}=\left\{y \in L \mid \lambda_{j}\left(\operatorname{proj}_{e^{\perp}}(y)\right)+\left(y \cdot e /\|e\|^{2}\right) \leq 0\right\} .
$$

Let $A$ be the linear mapping from $e^{\perp} \times \mathbb{R}$ to $L$ defined by $A(s, t)=s-\frac{t}{\|e\|} e$. One easily checks that $Y_{j}=A\left(\operatorname{epi}\left(\|\mathrm{e}\| \lambda_{\mathrm{j}}\right)\right)$, where epi $\left(\|\mathrm{e}\| \lambda_{\mathrm{j}}\right)$ is the epigraph of the function $\|e\| \lambda_{j}$, that is epi $\left(\|\mathrm{e}\| \lambda_{\mathrm{j}}\right)=\left\{(\mathrm{s}, \mathrm{t}) \in \mathrm{e}^{\perp} \times \mathbb{R} \mid \mathrm{t} \geq\|\mathrm{e}\| \lambda_{\mathrm{j}}(\mathrm{s})\right\}$. The mapping $A: e^{\perp} \times \mathbb{R} \rightarrow L$ is an isometry if $\|(s, t)\|=\sqrt{\|s\|^{2}+t^{2}}$ is the Euclidean norm on $e^{\perp} \times \mathbb{R}$. Hence, for all $(s, t) \in \operatorname{epi}\left(\|\mathrm{e}\| \lambda_{\mathrm{j}}\right), \perp_{Y_{j}}(A(s, t))=A\left(\perp_{\mathrm{epi}\left(\|\mathrm{e}\| \lambda_{\mathrm{j}}\right)}(s, t)\right)$, which implies $N_{Y_{j}}\left(A(s, t)=A\left(N_{\mathrm{epi}\left(\|\mathrm{e}\| \lambda_{\mathrm{j}}\right)}(s, t)\right)\right.$. Furthermore, from Corollary of Theorem 2.4.9 in Clarke (1983), we have:

$$
\partial\left(\|e\| \lambda_{j}\right)(s)=\left\{p \in e^{\perp} \mid(p,-1) \in N_{\mathrm{epi}\left(\|\mathrm{e}\| \lambda_{\mathrm{j}}\right)}\left(s,\|e\| \lambda_{j}(s)\right)\right\} .
$$


From these two results, one deduces that:

$$
\partial\left(\|e\| \lambda_{j}\right)(s)=\left\{p \in e^{\perp} \mid p+\frac{1}{\|e\|} e \in N_{Y_{j}}\left(\Lambda_{j}(s)\right)\right\} .
$$

Noticing that $\partial \lambda_{j}(s)=\frac{1}{\|e\|} \partial\left(\|e\| \lambda_{j}\right)(s)$ and $N_{Y_{j}}\left(\Lambda_{j}(s)\right) \subset P$ from the free-disposal, one deduces that:

$$
\partial \lambda_{j}(s)=N_{Y_{j}}\left(\Lambda_{j}(s)\right) \cap S-\left\{\left(1 /\|e\|^{2}\right) e\right\} .
$$

Finally, the correspondence $\partial \lambda_{j}$, from $e^{\perp}$ into itself, is upper hemi-continuous with non-empty, convex, compact values since $\lambda_{j}$ is Lipschitz continuous [see Proposition 2.1.2 in Clarke (1983)].

Proof of Lemma 5: From Lemmata 3.1 and 3.2 in Bonnisseau and Cornet (1990b), under the assumptions of Lemma 2, there exists an open neighborhood $\Omega$ of $M_{a b}$, two positive real numbers $\alpha, \nu$ and an infinitely differentiable mapping $F: \Omega \rightarrow E$ such that:

$$
0<\alpha<\inf F(s) \cdot \Delta\left(s^{\prime}\right) \text { for all } s, s^{\prime} \in \Omega \text { satisfying }\left\|s-s^{\prime}\right\| \leq \nu .
$$

Moreover, for every $s \in \Omega$, if $\xi(s, \cdot)$ denotes the maximal solution of

$$
\dot{s}(t)=F(s(t)), s(0)=s,
$$

and $I(s)$ the maximal interval of definition of $\xi(s, \cdot)$, then $\xi(s, \cdot)$ is a $C^{\infty}$ mapping from $I(s)$ to $\Omega$, and there exists a compact neighborhood $K \subset \Omega$ of $M_{a b}$ and a Lipschitz continuous function $\tau: K \rightarrow \mathbb{R}$ such that, for every $s \in K$ :

$$
\begin{array}{r}
\tau(s) \in I(s) \text { and } 0<-\tau(s)<|a-\theta(s)| / \alpha, \\
\theta(\xi(s, \tau(s)))=a .
\end{array}
$$

Since $M_{a} \subset \operatorname{int} \mathcal{M} \subset M_{b}$, we have $\partial \mathcal{M} \subset K$ and we can define the mapping $H: \partial \mathcal{M} \times[0,1] \rightarrow E$ as follows:

$$
H(s, t)= \begin{cases}(s-\xi(s, t \tau(s))) / t & \text { if } t \neq 0 \\ -\tau(s) F(s) & \text { if } t=0 .\end{cases}
$$

$H$ is clearly continuous since $\xi$ is the solution of the differential equation. For every $(s, t) \in \partial \mathcal{M} \times[0,1]:$

(a) $\inf H(s, 0) \cdot \Delta(s)>0$;

(b') $H(s, 1) \in-\operatorname{int} T_{\mathcal{M}}(s)$;

(c) $H(s, t) \neq 0$. 
Indeed, for every $s \in \partial \mathcal{M}, H(s, 0)=-\tau(s) F(s)$. Therefore, from (16) and (17),

$$
\inf H(s, 0) \cdot \Delta(s)=-\tau(s) \inf F(s) \cdot \Delta(s)>0 .
$$

Moreover, for every $s \in \partial \mathcal{M}, H(s, 1)=s-\xi(s, \tau(s))$. But, from (18), $\xi(s, \tau(s)) \in$ $\partial M_{a} \subset \operatorname{int} \mathcal{M}$. Since $\mathcal{M}$ is a closed, convex subset of $E$ and $s \in \partial \mathcal{M}$, we then deduce that $H(s, 1) \in-\operatorname{int} T_{\mathcal{M}}(s)$.

Let us finally show that $H(s, t) \neq 0$ for every $(s, t) \in \partial \mathcal{M} \times[0,1]$. From (16), this is true for $t=0$. Now, from (16), the mean-value Theorem and the fact that $\partial \theta(s) \subset \Delta(s)$, one deduces that $\theta(\xi(s, t))$ is a strictly increasing function of $t$. Consequently, $s=\xi(s, 0) \neq \xi(s, t \tau(s))$ for all $t>0$, which implies that $H(s, t) \neq 0$.

Proof of Lemma 6: For every $\nu \in \mathbb{N}^{*}$, we have $\tau^{\nu} \leq-1 / \nu<0$. If the sequence $\left(\tau^{\nu}\right)$ does not converges to 0 , then there exists $\varepsilon>0$ and a strictly increasing sequence of natural numbers $\left(\nu_{\mu}\right)$ such that $\tau^{\nu_{\mu}}<-\varepsilon$ for every $\mu \in \mathbb{N}$. For $\mu$ large enough, $1 / \nu_{\mu}<\varepsilon$ implies that $\min _{1 \leq i \leq m}\left\{\inf r_{i}\left(A P E^{\nu_{\mu}}\right)-\inf p \cdot X_{i}\right\}<-\varepsilon$, and there exists a consumer $i^{\nu_{\mu}}$ and an element $\left(p^{\nu_{\mu}},\left(y_{j}^{\nu_{\mu}}\right) \in A P E^{\nu_{\mu}}\right.$ such that $r_{i^{\nu \mu}}\left(p^{\nu_{\mu}},\left(y_{j}^{\nu_{\mu}}\right)\right)-\inf p^{\nu_{\mu}} \cdot X_{i^{\nu} \mu}<-\varepsilon$. Since, for $\mu$ large enough, $A P E^{\nu_{\mu}}$ is a subset of $S \times A_{t_{0}}$, which is compact under Assumptions (P), (C) and (BLS') or (BS') if $\varphi=M P$, we can suppose without any loss of generality that the sequence $\left(i^{\nu_{\mu}}\right)_{\mu}$ is constant, say equal to $i$, and that the sequence $\left(p^{\nu_{\mu}},\left(y_{j}^{\nu_{\mu}}\right)\right)$ converges to some element $\left(p,\left(y_{j}\right)\right) \in A P E$. From the continuity of $r_{i}$ and the upper semi-continuity of $p \rightarrow \inf p \cdot X_{i}$, passing to the limit in $r_{i}\left(p^{\nu_{\mu}},\left(y_{j}^{\nu_{\mu}}\right)\right)-\inf \tilde{p}^{\nu_{\mu}} \cdot X_{i}<-\varepsilon$, we finally get $r_{i}\left(p,\left(y_{j}\right)\right)<\inf p \cdot X_{i}$, a contradiction with Assumption (R').

\section{References}

Beato, P., Mas-Colell, A., 1985. On marginal cost pricing with given taxsubsidy rules. Journal of Economic Theory 37 (2), 356-365.

Berge, C., 1959. Espaces topologiques: Fonctions multivoques. Collection Universitaire de Mathématiques, Vol. III. Dunod, Paris.

Bonnisseau, J.-M., 1992. Existence of equilibria in the presence of increasing returns: a synthesis. Journal of Mathematical Economics 21 (5), 441-452.

Bonnisseau, J.-M., Cornet, B., 1988. Existence of equilibria when firms follow bounded losses pricing rules. Journal of Mathematical Economics 17 (2-3), 119-147.

Bonnisseau, J.-M., Cornet, B., 1990a. Existence of marginal cost pricing equilibria: the nonsmooth case. International Economic Review 31 (3), 685-708.

Bonnisseau, J.-M., Cornet, B., 1990b. Fixed-point theorems and morse's lemma for lipschitzian functions. Journal of Mathematical Analysis and Applications 146 (2), 318-332.

Bonnisseau, J.-M., Cornet, B., 1991. General equilibrium theory with increasing returns: the existence problem. In: Equilibrium theory and applications 
(Louvain-la-Neuve, 1989). International Symposium in Economic Theory and Econometrics. Cambridge University Press, Cambridge, pp. 65-82.

Clarke, F. H., 1975. Generalized gradients and applications. Transactions of the American Mathematical Society 205, 247-262.

Clarke, F. H., 1983. Optimization and nonsmooth analysis. Canadian Mathematical Society Series of Monographs and Advanced Texts. John Wiley \& Sons Inc., New York, a Wiley-Interscience Publication.

Cornet, B., 1989. Existence of equilibria in economies with increasing returns. In: Contributions to operations research and economics (Louvain-la-Neuve, 1987). MIT Press, Cambridge, MA, pp. 79-97.

Debreu, G., 1956. Market equilibrium. Proceedings of the National Academy of Sciences of the United States of America 42, 876-878.

Debreu, G., 1959. Theory of value: an axiomatic analysis of economic equilibrium. Cowles Foundation for Research in Economics at Yale University, Monograph 17. John Wiley \& Sons Inc., New York.

Debreu, G., 1962. New concepts and techniques for equilibrium analysis. International Economic Review 3, 257-273.

Dehez, P., Drèze, J., 1988a. Competitive equilibria with quantity-taking producers and increasing returns to scale. Journal of Mathematical Economics 17 (2-3), 209-230.

Dehez, P., Drèze, J., 1988b. Distributive production sets and equilibria with increasing returns. Journal of Mathematical Economics 17 (2-3), 231-248.

Dehez, P., Drèze, J. H., Suzuki, T., 2003. Imperfect competition à la Negishi, also with fixed costs. Journal of Mathematical Economics 39 (3-4), 219237, special issue on the Athens-Minnesota Conferences (Minneapolis, MN/Athens, 2002).

Dierker, E., Guesnerie, R., Neuefeind, W., 1985. General equilibrium when some firms follow special pricing rules. Econometrica 53 (6), 1369-1393.

Gale, D., 1955. The law of supply and demand. Mathematica Scandinavica 3, 155-169.

Guesnerie, R., 1975. Pareto optimality in non-convex economies. Econometrica $43,1-29$.

Hotelling, H., 1938. The general welfare in relation to problems of taxation and of railway and utility rates. Econometrica VI, 242-269.

Kamiya, K., 1988. On the survival assumption in marginal (cost) pricing. Journal of Mathematical Economics 17 (2-3), 261-273.

Nikaidô, H., 1956. On the classical multilateral exchange problem. Metroeconomica 8, 135-145.

Rockafellar, R. T., 1970. Convex analysis. Princeton Mathematical Series, No. 28. Princeton University Press, Princeton, N.J. 